\title{
Second Screen Engagement of Event Spectators
}

\author{
Tomas Cerny (iD) and Michael Jeff Donahoo \\ Computer Science, Baylor University, Hankamer Academic Building, No. 105, Waco, TX 76706, USA
}

Correspondence should be addressed to Tomas Cerny; tomas_cerny@baylor.edu

Received 3 May 2018; Accepted 25 June 2018; Published 10 July 2018

Academic Editor: Hideyuki Nakanishi

Copyright (C) 2018 Tomas Cerny and Michael Jeff Donahoo. This is an open access article distributed under the Creative Commons Attribution License, which permits unrestricted use, distribution, and reproduction in any medium, provided the original work is properly cited.

\begin{abstract}
An effective means of engaging spectators at live events involves providing real-time information from a variety of sources. Consumers demand personalized experience; thus, a single channel perspective fails. Modern entertainment must extend to spectator mobile devices and adapt content to individual interests. Moreover, such systems should take advantage of venue screens to engage in sharing live information, aggregated social media, etc. We propose a second screen application, providing each audience member a personalized perspective, involving mobile devices equipped with $\mathrm{Wi}$ - $\mathrm{Fi}$, and spanning to venue screens in hotels, halls, arenas, elevators, etc. Such a system engages both local audience and remote spectators. Our work provides a case study involving experience from the deployment of such an application at the ACM-ICPC World Finals with audiences at the event and around the world. We analyze and categorize its features, consider its impact on the audience, and measure its demands.
\end{abstract}

\section{Introduction}

Sporting matches, conferences, cultural events, elections, debates, concerts, and other live events bring opportunities for real-time engagement of the audience. The traditional approach emphases a single stream of information, usually a television broadcast, with a single source of commentary. The sequence of actions in the broadcast program cannot accommodate a multidimensional perspective; thus, the broadcast might not fully engage the audience, thereby degrading the Quality of Experience (QoE). Such an approach is outdated in the era of on-demand service; [1] proposes augmenting the mainstream of information with Second Screens.

Second Screen is a display (e.g., smartphone, tablet, and smartwatch) designed to augment primary viewing by providing additional content. Such devices are typically mobile and interactive, allowing content individualization. Examples include augmenting live sporting events with detailed statistics on selected players, broadcast television with social engagement, etc.

It is important to engage the audience and give an option to participate in the process of information broadcast. Such an extension makes the event more authentic for the audience. Moreover, the audience can express and share its own perspective to others. Furthermore, engaging local audience members in sharing their experience promotes their view to the overall audience, possibly expanding the reach to new followers. There is no single view in Second Screen; users choose the individual perspective to follow, e.g., following the live event, digging into detailed information, seeing updated statistics, etc. An event host should consider Second Screen to provide the audience with wide-angle perspectives full of extensive and easy-to-reach information. Such services power the audience with authentic entertainment impacting the QoE [1].

The personalization and context-awareness [2] might be further extended when considering a cosmopolitan audience with different cultural backgrounds, habits, or pleasure. A single information channel can only partially satisfy such audience. The Second Screen augments the program and extends the individual experience.

Present-day event participants usually possess mobile devices capable of presenting Second Screen services, opening Second Screen interaction to the overall entertainment environment [3]. Furthermore, nowadays event arenas usually provide large screens that can become part of the Second Screen experience by providing a content influenced by the crowd. 
Integrating social media further engage users in the new role of mediators that can influence the crowd and crowd experience. This provides a way to share authentic information from a personal perspective, avoiding single (professional) perspective information since all participants comment and judge.

Here we share our experience from analysis, design, and production-level deployment of a Second Screen system, MyICPC, applied to the ACM-ICPC World Finals. As described later, this event involves over 1,000 local and over 10,000 remote participants from 115 countries. A variety of services are provided for the heterogeneous audience, and this article addresses audience demands and reveals which services are the most important, based on our case study. The generalized problem being addressed is the monotony of traditional, passive spectating, and our view on its transformation towards future entertainment systems.

While we do not provide an evaluation of usability or user experience, we show that local audience uses Second Screen to augment the event experience. We explore particular factors that impact audience interest in the service utility [4]. Based on the motivational factors, observations and suggestions researched in this paper, we implement a productionlevel Second Screen application. The application has been deployed for several years and accessed by a large audience, i.e., tens of thousands of users. We study the audience interest and utilization of our Second Screen application by both local and remote audience involving various device types.

This article is organized as follows. Section 2 provides a survey on related work on Second Screen systems. Section 3 gives background and analysis of information sources or screens available for services in the venue. Section 4 moves the attention towards architectural decisions and system design. A case study sharing practical experience from deployment is in Section 5. The conclusion and future work close the paper.

\section{Related Work}

Considerable works from a variety of perspectives exist for Second Screen [3-14]. The applicability and research goals can be classified by the target audience, considering both local and remote users. We divide related work into multiple subsections. First, we consider Second Screen services for entertainment such as movies or event reproduction for remote users. Next, discuss the scope of Second Screen applications. The social media subsection discusses the importance for the audience. Next, we elaborate Second Screen engagement in live events. Finally, we address extending spectator experience.

2.1. Passive Entertainment Reproduction Services. When considering passive entertainment, researchers address user's QoE in a home environment using multiple devices augmenting the main TV screen $[3,5,8,10]$. Research goals pay attention to video broadcasting and network requirements for high-quality streaming of on-demand video [1]. The passive home consumer of entertainment can become involved through social networks by sharing the experience
[13]. For instance, [3] considers that consumers would discuss their movie experience over chat. Second Screen augmenting traditional TV programming can bring a novel dimension to sports events with an overlapping program, which is usually at large events such as the Olympic Games. The authors [5] evaluate the use of the Olympic Companion application, collocating viewers to follow multiple simultaneous channels of sports events providing statistics and allowing switching them between different devices or move in time.

The study [3], performed in a home environment, involved 260 tablet users, investigating how users incorporate multiple media into their television viewing experience. The results indicate only a modest interest in using secondary screens to digitally share opinions. Naturally, the engagement of such users is not expected to be significant since they are only receiving and consuming the entertainment experience, specifically movies. One must consider that users paying attention to movie plot on the main TV screen do not necessarily demand interaction. When considering an interactive environment, the outcome is different. The interpretation of such results cannot simply generalize to all environments or to Second Screens. For instance, [4] suggests that environments involving active spectators create an authentic social event atmosphere that motivates spectators to attend the event and share the experience with others over Second Screen. The Second Screen is also often used to complement the information provided on the TV screen [12].

A report on a many-screen prototype application for watching the Olympics, provided by [5], examines users engaging in a parallel view to multiple simultaneous channels and integration with multiple Second Screen devices. Users can watch, share, and control highlight programs. In addition, they can obtain event statistics or replay portions of the broadcast. While users can watch multiple devices at once, the authors noted that users become confused and struggle to maintain comprehension of liveness. The benefit is that users select content based on their interest, even when there is overlap.

The papers [6] and [9] provide a roadmap to the various applications and features of Second Screen. Its applicability to the video content interaction involves replays, playback point selection, or gestures to simplify interaction with the content. It can bring interaction with social media or deliver contextual information for the television content. For sporting events, Second Screen usually gives statistics (e.g., about sports players) to augment the event with related information. Second Screens can mediate these events and provide a better experience to the home audience with combined interpretation and more authentic narrative structure.

2.2. Scope of Second Screens. There is no strict boundary that would distinguish Second Screens from computer-aided assistant-like applications. For instance, consider a situation of a system similar to Second Screen that helps users to accomplish certain tasks, e.g., when a visitor aims to navigate through a large complex hospital. The hospital has its own navigation system, but for a person with health/emotional challenges, the navigation might become difficult. A personal smartphone used for in-house navigation [15] provides a sort 
of Second Screen. Furthermore, the approach can easily adapt to visitors based on their age or disabilities.

Such systems have a different focus than Second Screen. For Second Screens, the aim is to improve user experience, and the other computer-aided system example aims to assist the user with a particular task. Second Screen is meant as an augmentation system related to a live event or a stream of information, while the computer-aided assistant is a more general, on-demand service focused on individual need or specific task instead of a social event. To draw a distinctive example, consider a smartphone hospital navigation system providing social media service. Such an integration is inappropriate. On the other hand, integration to the Second Screen is a good fit.

2.3. Social Media. Previously, we mentioned interaction through social media. In particular, when considering active spectators, we might expect their active involvement in the mediation of the authentic experience through social media [4]. Most importantly the crowd not only generates but also evaluates the authenticity and thus avoids subjective perspective, given by traditional mass media. Naturally, Second Screens should provide the ability to integrate social media interaction. Moreover, social media integration should be an integral part of Second Screens.

The role of social media and networking is elaborated in [3]. Nowadays it competes with the traditional perspective of mass media. Undoubtedly, mass media can bring a subjective perspective that is falsely consumed by the audience. Social media provides a counterpoint for such opinions since all active participants share real-life and instant experience and judge opinions of others. A contemporary audience equipped with mobile devices participates in media crowd sharing through a variety of channels [16]. This changes the traditional perspective of living with media into living in media [17]. The technological growth creates the basis for multimodal, self-generated, self-directed, and self-selected mass media communication [18]. From such a perspective, the Second Screen seems to have all the prerequisites to become an instrument to share experience and produce media and public opinion. The Second Screen should stimulate the audience interest in publishing personal perspectives [7]. Multiple works consider Second Screens with social media integration as a good instrument to receive public opinion [14]. In [11] the authors analyzed tweets that were published related to TV broadcast and categorize them by their purpose, such as opinions, interpretations, emotions, attention seeking, or sharing information.

2.4. Second Screen at Live Events. When considering live events, the audience divides into active and remote spectators. The active spectators follow the central perspective of the event and may use Second Screen to augment their event experience using personal devices, venue screens, projectors, etc. For remote spectators, Second Screen may act as the central perspective of the event or become the primary source of information. Moreover, both groups may interact through Second Screen; active spectators contribute to the crowds through social media and mediate the authentic experience from the event to others.
Research involving Second Screen at live events aims to bring a new dimension of experience for active spectators or audience. For instance, the Second Screen application can accompany live performances in theatres [6] or impact experience for active spectators in large sporting events [4]. Moreover, Second Screens are applied in museums and galleries [19]; their deployment takes place at concerts [4], allowing the performer to interact with the audience to extend the social impact on the passive audience, while bringing opportunities for public opinion or voting, etc.

Second Screens may find its role at places that would traditionally deny the use of any distracting technological device. Live performances can take advantage of Second Screen either from the perspective of media sharing or extra content. When considering video streaming in such events, the multiangle perspectives, zooming, or playback point selection abilities potentially improve audience experience [6]. While previous research shows a certain issue comprehending with liveness [5] or passivity in Second Screen usage [3], the outcome from Second Screen usage in live performance, experienced in [6], shows that the audience is able to keep control over their viewing experiences through the Second Screen video feed while maintaining a presence in the live performance.

Applicability to live sporting events is researched by [4]. Their Active Spectator, as applied to football matches, aims to improve spectator experience. The expected spectator is an active participant (a local audience), creating the atmosphere of the entire event. Their highlighted feature is the ability to access player's biometrical information as well as showing statistics. Note that conventional technology in the arena already employs some Second Screen-like features. For instance, large displays at sporting events augment with local experience, with an overlay resembling and, to some extent, simulating watching the event on television. On the other hand, such features could become available on-demand when involving individual's mobile phone, allowing a replay or selection of available options. Second Screen features might be inspired by such coexisting elements.

2.5. Spectator Experience Model. What convinces individuals to attend such events? The authors [4] suggest that the motivating element for the live event seems to be the social atmosphere at the sporting arena. To spectators, it means a great deal to be part of a large group having a social experience. Their identification and socializing with other spectators are perhaps the crucial factor in their engagement with the event. The personal experience is certainly more than a passive consumption of the sport itself. Specifically, an active attendance impacts the social aspect. There are three main aspects that can be taken into account: the sport itself, the event, and social aspects.

To constitute spectator's experience at sporting events and explore the potential of interactive technologies for active spectating, [4] define spectator's experience model (SPEX). The model opens up a broader perspective on the entire spectator experience and follows the three aspects of sport, event, and social. The SPEX model is a good instrument to provide a base for Second Screen services. Consider 


\begin{tabular}{|c|c|c|}
\hline \multicolumn{3}{|l|}{ Social } \\
\hline \multicolumn{3}{|c|}{$\begin{array}{l}\text { Attending with friends; Experience from events; } \\
\text { Collective solidarity; }\end{array}$} \\
\hline Sharing stories & Self-presenta & $\begin{array}{l}\text { ation; Traditions; } \\
\text { Spectators battle; }\end{array}$ \\
\hline $\begin{array}{l}\text { Fan-culture; } \\
\text { Identification }\end{array}$ & $\begin{array}{c}\text { Instruments; } \\
\text { Merchandise; } \\
\text { Cheering }\end{array}$ & $\begin{array}{l}\text { Activities before } \\
\text { /after the event; } \\
\text { Special guests }\end{array}$ \\
\hline $\begin{array}{l}\text { Team history; Rules; } \\
\text { Statistical data; } \\
\text { In-game events }\end{array}$ & $\begin{array}{l}\text { Program; } \\
\text { Activities }\end{array}$ & $\begin{array}{l}\text { Food \& drinks; } \\
\text { Venue; } \\
\text { Location }\end{array}$ \\
\hline
\end{tabular}

Discipline / sport

Figure 1: The SPEX Model elements constituting the spectator experience.

a generalized SPEX model illustrated on Figure 1. The sport, or a particular discipline, has specific rules, but most likely it also has a history, statistics, and perhaps specific events. Furthermore, there might be related activities, identification with other fans, and overall a culture of fans. This all might involve special forms of cheering teams, support certain merchandise, or suggest some specific instruments supporting identification. The social part may involve stories and interaction with fans, as well as collective solidarity. The event should give spectators a chance to present themselves, follow certain traditions, support event specific activities, or compete with other spectator groups.

2.6. Human Factors: Lurking. Important aspects relevant to effectiveness of Second Screens can be found in research on human factors $[20,21]$. It is known that online learning develops through interaction and it is a collaborative process engaging learners actively. However, there is an effect, known as lurking impacting the passive audience, which is relevant to Second Screens. According to [21], up to $90 \%$ of online discussion group participants are lurkers. Lurkers are an audience that does not actively contribute to the content and do not produce a post. In [20], the authors consider elearning and discuss the voluntary participation in virtual interaction to improve learning and consider lurking, evaluating passive participation. In the learning context "lurkers are learners who are bystanders to course discussions". They lack commitment to the community; however, they receive benefits without giving anything back. This is one explanatory factor for the effectiveness of Second Screens. In [20], the authors conclude that a higher degree of visible interactions is not a precondition for higher learning efficiency and lurking must be considered in this activity. The passive lurking is expressed by reading and reflecting on the contribution of all the participants.

\section{Background and Analysis of Second Screen Applications}

For our analysis, we begin by first considering the physical setup both before and after Second Screen deploy. Next, we take into account the local and remote audience or the event information sources. Finally, we evaluate the services and features that improve the experience using the SPEX model, generalizing the sports aspect as a discipline.

The physical setup of events is usually as follows. The event is situated in an arena; it can be a stadium, hotel, conference hall, resort, etc. Such an arena might have restricted zones, such as VIP, secure, and visitors. Each arena has a set of resources such as stages, large announcement screens, projectors and TV screens in hallways, elevators, and possibly hotel rooms. Spectators may (or may not) have a personal device such a phone, tablet, and personal computer. Each arena provides at least access to some mobile network and perhaps even high-density access points with Wi-Fi.

The physical setup for the remote audience is often much simpler. The audience follows a website, live stream video broadcast, or social network from a public place such as school, pub, square, or a private place such as a home. The physical setup includes TV screen or access to the Internet with a browsing device.

3.1. An Event without Second Screen. The information sources of a live event correspond to its schedule. The schedule is most likely available on a centralized website, so the main website and the schedule itself are the most critical parts of information sources before the event starts. The schedule gives an overview and roadmap to subevents and parallel sessions or stages. There may exist online preevents in which the audience might participate. At this point, audience interaction and their grouping are expected through social networks, so supportive feeds or platforms, such as Facebook, LinkedIn, Twitter, Instagram, YouTube, and VKontakte, are expected to involve the crowd. The website may provide up-to-date statistics, speculations, opinions, and perhaps remarks from the last event.

Once the event starts, we may see the distinction between local and remote audience. Regarding the local audience, the old fashion approach might involve program printouts handed to attendees who themselves follow a static schedule. This may involve arena screens, showing the actual schedule information. In this approach, we do not receive any feedback from the audience nor support their social involvement. Most likely the audience takes pictures of the venue and posts them on their personal social profile. The missing piece is the centralization and online socializing with other attendees. In this approach, each attendee posting media is to be rather isolated. Even if we consider, for instance, posting a Twitter message following a certain hashtag, how do we connect Twitter users with VKontakte users, Facebook users, etc.?

From another perspective, the event has a well-defined schedule, professional mediator, and media team providing pictures and video from the event to the audience through large arena screens or managing a scoreboard. Here the audience is not engaged. What if a random participant takes the best photograph from the world championship? Such a photograph may have limited public exposure. Furthermore, specific participant interests are not highlighted in traditional mechanisms or instruments. Thus, in order to follow specific 
aspects of the event, each participant must assemble relevant particles himself/herself.

We often see these deficiencies in traditional local participation with a static schedule that does not adapt to what the crowds likes. Next, it does not involve the audience in public opinion, self-presentation, feedback to certain subevent, and social network interaction during the event among various participants. The missing piece is a centralized source of information involving professionally made content, event results, audience-generated content, etc., all allowing personalization, real-time event processing, and interaction.

The remote audience can follow a TV broadcast, blogger, Twitter feed, or the event website. The deficiency is similar to local participation, missing a centralized source of information supporting crowd interaction, and possibly missing an important comment or photograph from the event.

After the event, the media team provides short videos, blogs, photographs, statistics, and perhaps other articles and event mentions, accessible through the website. To some extent, the event website may act as the central place for information, although integration of other third-party information sources is limited. The website plays the main role when publishing information, both before and after the event. During the event, the website does not play a significant role. The audience attention flees in all directions. For instance, the audience follows the event performance, social interaction at third-party providers, the environment, various social media, specific blogs, etc.

Figure 2 draws a simplified interaction between information sources, information destination, its mediators, and consumers. The media team has access to the environment. The team has access to live event information both the public side and internal information that it interprets and mediates to the audience. The team then provides such internal or private information to the website, blogs, or social media. The local audience has access to public live events and, in addition, the social interaction in the environment. When the audience interacts with other sources of information, it might be relevant to custom social media services, blogs, or even the website. Thus, the audience may produce content of broad interest in a distinct space such that it never reaches other members of the audience. The passive audience is the consumer of the outcome produced by the media team and local audience.

3.2. An Event with Second Screen. The Second Screen platform should target a change in the interaction from distributed to a centralized destination. Similar to the situation before and after, the Second Screen platform should act as the website and thus be web accessible. Next, for the live event, the Second Screen platform should centralize contributions from social networks, blogs, and media teams, so that the audience has a single focal point to observe and receive all the information. The transition to the proposal is illustrated in Figure 3.

So far we only analyzed and suggested a centralized view on the interaction among information sources, audience, and their contribution. We did not analyze the features and the possible utility of Second Screen, although centralization is

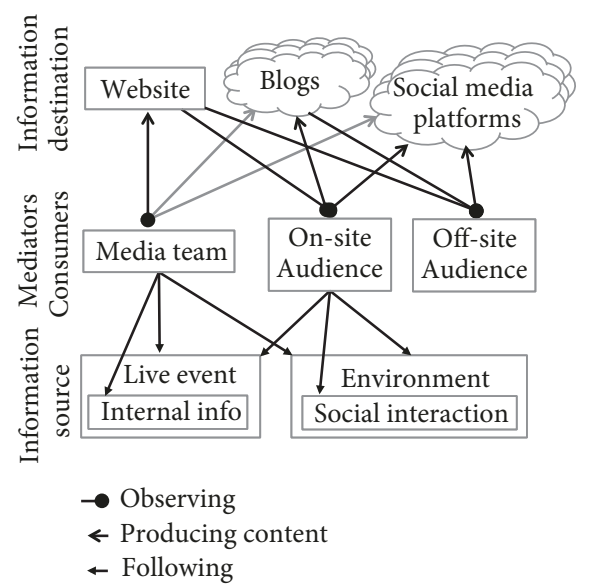

Figure 2: Emitting information (traditional env.).

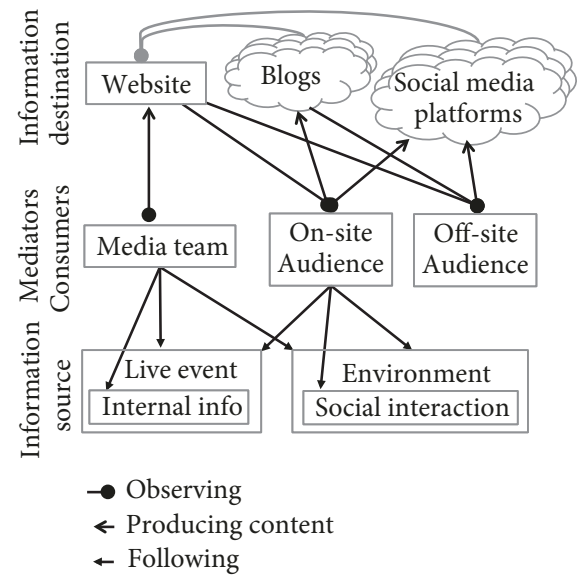

FIGURE 3: Emitting information (Second Screen env.).

an important step as it allows us to provide a custom, contextaware view to an individual. The SPEX model suggests that the Second Screen platform considers various aspects. It should consider the discipline and provide statistics of players' team or actors, or even past events. The schedule is a naturally important element as well as rules all in a structured format so that it is easy to link it to statistics with currently scoring player or performing actor or even location in the venue.

To increase motivation to attend the event, the audience should have the ability to present them. This could be an ability to register their profile, post comments, pictures, and video, or be involved in social media showed on Second Screen. Receiving reaction to their contribution, such as comments, can socialize fans that may normally not meet in person. Furthermore, the public feedback can boost motivation for the audience to contribute with a personal perspective and view. The audience can cheer for their favorite team or actor, and the sponsors or organizers can involve the audience in social quizzes or puzzles to recognize the winner at the event. 
The Second Screen should provide the mainstream information on the event. It can involve video broadcast, active scoreboard, and an ability to quickly list details or highlight certain perspectives, angles, or aspects. The crowd in the social network can react to polls from organizers; see statistics on what the crowd likes or how many participants are following a particular team.

To integrate large screens in the arena, venue, hotel, etc., the Second Screen should not only consider a static schedulelike content but also involve live information from the crowd. In particular, the crowd posts a large number of social media and messages reflecting the scenery. The large screens should display the real-time social media content to further engage the audience in involvement since individual's post appears on the screens. Since the crowd contributes to the content and interacts with the large screen content, the content corresponds to the current scene giving an authentic reproduction of the local audience scenery to the remote audience. At the same time, malicious posts must be filtered on an automated or a manual basis.

Certain events, such as conferences, can even connect the social contributors with their profile and possibly identify their media or professional profile such as LinkedIn. Media content, such as photographs, can be augmented with metainformation to be linked to the schedule and events. In [22], a gallery system recognizes participant faces in pictures. This process can be a semiautomated, involving user profile details with their picture involving automated face recognition in photographs captured at the event. The audience can list their personal gallery during or after the event. Furthermore, a broader media gallery can reconstitute the event with various details. Moreover, metainformation enables integration of media content across various features of Second Screen. For instance, a program event includes photographs from the event, teams are augmented with venue pictures, winning can be tracked with its history, or an awarded actor of the night may be seen the play from various angles when listing actor details.

The Second Screen is an ideal place for announcements for events such as free snacks at intermission or an interview with a famous person. The Second Screen should become the medium for discussion. For instance, consider a large competition with the sudden decision to disqualify a particular team. Judges can provide their rationale or respond to the public.

Next, consider mediators who speculate on who is going to take the lead in a competition or what not to miss in a particular performance. For instance, in a live performance or a concert, the audience is notified that it is about the time that critics have pointed out as the best part of the night, which can serve as a narrator or a guide to the show. The Second Screen should become an instrument to interact with the audience to receive their immediate feedback since; in live events, a two-minute delay might be too late to ask how they like a particular piece or action by the performer.

To summarize, Second Screen features should provide real-time, on-demand services and allow augmentation of the event. Moreover, it is an instrument for announcements as well as a dynamic schedule. It should be possible to access player details, annual statistics, upcoming events, the map of the environment, rules, or static content in a structural form to be able to associate the content with scoring players, etc. The Second Screen should also aggregate real-time statistics for augmenting the event. It should have the ability to present the current scene, for instance, a video broadcasting, scoreboard, interactive map, etc. Particular attention should be given to the simplicity of service integration, such as connecting a schedule and places with media pictures from the crowd, and every entry becomes interactive through social networks. All posts from blogs, social networks, media teams, judges, bloggers, etc. should be integrated into a chronological timeline. All posts, including audience media, should appear in a crowd-gallery involving metainformation to associate it with time, place, person, team, or social media account. These media can highlight related textual content when viewed by the audience.

\section{Design and Architecture}

The previous sections suggest that Second Screens interact with both remote and local users. What are the options when building Second Screens? Some solutions provide dedicated applications, specific to a given operating system, device, etc. A dedicated application takes advantage of platform-specific features in the user interface to support efficient interaction. There are disadvantages of such native applications. First, the user has to download and install it. Users are understandably resistant to installing new applications since they take up resources and may present security risks. Second, users that do not have a compatible device cannot use such Second Screen apps. While one can implement a version of the application for each platform, the development costs may be considerable. In addition, one must be willing to actively maintain such applications, particularly if they inadvertently introduce a security or system-stability flaw. Thus, for proofs-ofconcepts, the dedicated application options seem reasonable, but to interest large heterogeneous audience it might be better to consider a web application. Various device types such as phones, tablets, and personal computers, no matter the platform, can access a web application. Recent technologies available for web provide publish-subscribe mechanism, notifications, and large content streaming, which is sufficient for Second Screens.

Web applications usually involve centralized data, interacting with various sources such as social media and blogs, while having the ability to produce content in real-time. The presentation part of a Second Screen application should adapt to various devices such as mobile phones, tablets, personal computers, large screens, or even smartwatch. The presentation should be responsive, including adjusting to various screen sizes and asynchronously notifying the user in near real-time when new information is available.

At the same time, we must expect scaling ability with respect to the number of users. A large number of media resources can be involved, including images and videos possibly demanding for large storage. This may require balancing and routing to content delivery networks. The Second Screen must have access to the main event information to process 
them. Such information can involve video broadcasting, direct input from judges or directors, event feed from the internal control system, current scoreboard information, etc.

Second Screens should take advantages of existing frameworks involving enterprise architectures [23]. Such platforms involve multiple tiers, such as client, server, and database tiers. In order to minimize demands placed on the server tier, a significant portion of the computational efforts should be offloaded to client devices. However, we must take into account the fact that the client may be a smartphone with limited resources and abilities.

Our experimentation shows that technologies such as Meteor [24] or Google Web Toolkit [25] are good for interactive pages but do not fit all types of phones or even tablets. In our prototypes, some important pages have a $10+$ second load time due to limited hardware. The other extreme is a fully server-side page rendering such as JavaServer Faces [26], but such an approach is not appropriate for real-time applications. In our prototype evaluation, we found that a framework like AngularJS [27] allows incremental page additions to predefined templates and suits to standard nonincremental dynamic content situations.

The server tier is a 3-layer application [23] involving presentation, business, and persistence layer. The server tier requires elastic scalability; therefore, the server application must allow for dynamically expandable computational instances. Given this, the initial design of the application should consider employing Platform as a Service (PaaS) and enable its deployment to the cloud.

Some Second Screen server components must be specialized. For instance, only one instance of the application should send emails, parse input from social media, feeds, etc. and share it with others. One approach is to employ a master-slave architecture, where the master instance parses and broadcasts all new information through messaging system to other instances. Slave instance then through a full duplex communication (e.g., web-socket protocol) announces novel information to clients. Clients interpret the novel information and publish it in the user interface. Recent evolution in application servers, which aim to support PaaS, strips out the need for heterogeneity and introduces a singleton service in a cluster environment. Modern application servers interact in the cluster (e.g., through an Infinispan as suggested by [28]) and exchange information. This allows dynamically installing the singleton service to a single node in the cluster. When such node disappears or fails, the service relocates in the cluster to another node.

The server tier services should provide both HTML outputs and JSON format for dynamic information embedding. The Singleton Services involve parsers of various social media feeds, event feeds with actions from the event, and possibly other inputs. The mechanism to share information for various purposes may benefit from event-driven approaches and messaging systems. For instance, a Twitter message is parsed and announced to observers that check the message for inappropriate content, publish it on the timeline, check whether the content relates to a social competition, and finally, an observer checks the media attachment and adds it to the gallery. Each of these observers is independent to the announcer and other observers, which allows extensibility of services. An observer may push the received event through a messaging system, such as Redis [29], to other nodes in the cluster that further mediate it to the clients. A new client may request a page that either contains dynamic content that does not further change or that incrementally updates through the full duplex communication. When a client visits a page that incrementally updates, the web browser subscribes to listen for server announcements. Thus, once a message from a messaging system appears, the subscribed client directly announces a novel content. The advantage of coexisting systems involving social media is offloading the hosting of media files and thus delegating the responsibility of content management.

As mentioned previously, Second Screen should consider spanning its services to use indoor venue screens. The simplest solution is to involve a static schedule. An extended option is to update the schedule dynamically based on time. To the engaged audience, the application should consider displaying live announcements and notifications from social media and the crowd. For instance, a screen accommodates up to 16 latest messages and dynamically updates the oldest with new posts. Most likely Second Screen already processes various feeds and channels, such as Twitter, YouTube, Instagram, etc.; thus, when a message gets processed and passes all filters it displays on the screens. This brings motivation to the crowd to get involved and post individual messages via a variety of social channels. The motivation for an individual is to make his or her post visible on the screens and this interacts with the screen and the crowd. Such multisource integration satisfies a variety of contributes since they can use a different application to produce the message content. Moreover, it is a good place for system announcements. In order to control all screens, while having the connection to a web-based Second Screen, the administrator must be given the ability to temporarily change the screen content. Underneath each screen is a web browser using a full duplex communication or polling; thus, it is possible to send control messages directly from the Second Screen and enforce navigation to another page, etc.

Scalability is one of the most important quality attributes. Based on our performance experimentation, we provide suggestions based on our observations. There exists a standard design pattern to deal with transaction management called Open Session in View (OSIV) [30]. In OSIV, each time a request appears from the client tier, a new transaction opens to fetch data from the database by particular services, and the transaction is kept open until the request fully processes. This provides an elegant approach to designing application without manually opening and closing the transaction in the source code of each method, thereby reducing the volume of code while maintaining high readability. Unfortunately, there is a negative side of this pattern. This pattern is used with persistence frameworks to load and lazy-load data and their associated collections when necessary. The advantage is that the responsibility of a manually defined service is offloaded to a framework programmatically, which is convenient; however, we lose control over what data are being loaded and how they are loaded, which potentially introduces significant performance issue. It is a considerable difference 
from the performance perspective, to load a single object versus thousands of others, while not even being aware of it. Based on our experiments, while OSIV is fine for small audiences, it lacks large scalability so we recommend avoiding it.

Finally, the database tier can involve NoSQL, relational database, or even both. The target domain, features, and complexity of statistics provided by the Second Screen determines the specific decision. In the case of complex data manipulation and table joins, a relational database might suit better. Based on our experimentation, the relational database sustains the large load; furthermore, relational databases can be clustered or use a cache and data virtualization such as Teiid (http://teiid.jboss.org).

\section{Case Study}

Our case study focuses on the Second Screen for the World Finals of the International Collegiate Programming Contest (ICPC) where the top university students in computing compete. The annual contests are multitier where teams of three contestants from the same university are given a set of algorithmic problems. The team that solves the most problems in shortest time wins. Annually over forty-thousand students attend ICPC contests. The World Finals is the final round, taking place at a single, physical location.

The World Finals event takes five days with a five-hour contest on the last full day and a practice session on the previous day. The contest with $120+$ teams takes place at sports arenas or large cultural halls with all teams in one place. The students are selected from $100+$ countries worldwide to represent their university.

The venue provides high-speed $\mathrm{Wi}-\mathrm{Fi}$ and is equipped with screens in the lobby, hotel rooms, and large projectors in the contest room. While students compete in designated area, they are not allowed to use any personal devices or access the Internet during the contest or practice session. On the other hand, spectators are free to use any personal device. Before and after the contest/practice, everybody, including contestants, staff, coaches, and spectators (both on and offsite ), can access mobile devices during the entire set of days.

The contest staff has many distinct groups such as judges and system operations producing various information streams such as contest scoreboard, media (video, photo, and blog teams), or analytics to augment the scoreboard and evaluating teams, etc. Some of the staff are responsible for publishing information on social media networks, blogs, picture galleries, video feed, etc.

In 2013 an initial beta version of Second Screen called MyICPC was introduced, integrating information sources such as social media, scoreboard, analytics, and media. The 2014 full version extended the MyICPC with statistics for teams, contestants, or contest attendees and introduced a social challenge called Quest. The rationale behind Quest is to socially engage attendees with activities during the five days. Quest gives multiple tasks (Figures 4 and 5) to the local audience who individually post through social media. Questers are posting pictures and video on Twitter and Instagram. The MyICPC provides polls to solicit public opinion. The major

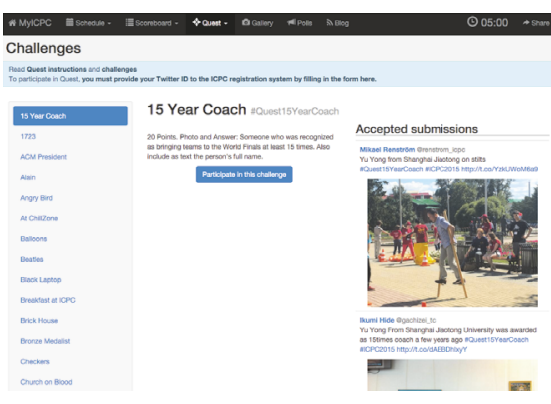

FIGURE 4: Quest challenges.

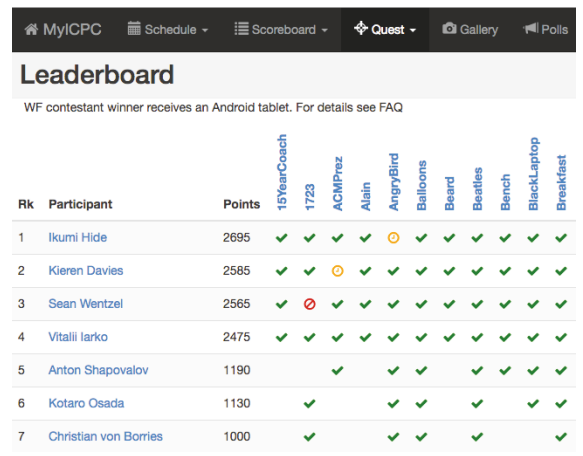

Figure 5: Quest leaderboard.

element of the MyICPC is the master timeline (Figure 6) that integrates all events in a sequence feed and updates in realtime. Users can follow the schedule with highlighted current time and a map (Figure 7). A gallery (Figure 9) integrates social media, photos, and video both from the professional media team and the crowd. The media team posts pictures with metadata in it as mentioned in [22], link media content with a particular team to the team's statistics (Figure 13), places in the venue, events in the schedule filtered by person, team, or event.

To push MyICPC to the venue screens, a special information kiosk is provided. This kiosk integrates a variety of channels and feeds and shows texts, images, and videos from the audience on the screens. This highlights the current activities on social networks, presents the current schedule, or emphasizes notice. In our setup, the kiosk was presented on screens during registrations, dinners, and other social events. Sample kiosk contents are provided in Figures 17 and 18.

To accommodate all variations of screen size, MyICPC uses responsive user interfaces design. The content adjusts for a range of screen formats. For example, Figure 10 shows the layout on a large computer screen, while Figure 11 demonstrates the tablet layout. AngularJS offloads certain computations to clients, while not demanding enormous resources. Each user can follow preferred content, such as particular team candidates that are highlighted when relevant event occurs (Figure 10). Users can specifically follow the rank in their region by following a regional map (Figure 8). The team perspective (Figure 14) provides live updates on accepted and rejected submissions, rank, passed test cases, 


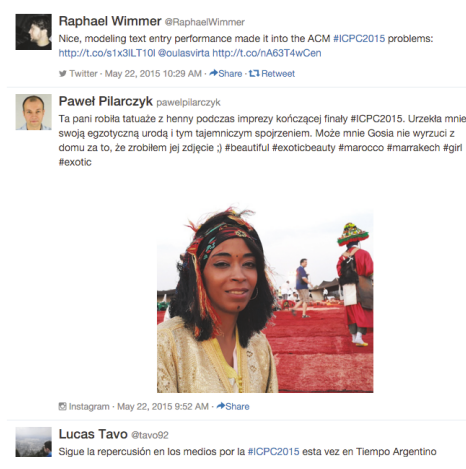

Figure 6: MyICPC master timeline.

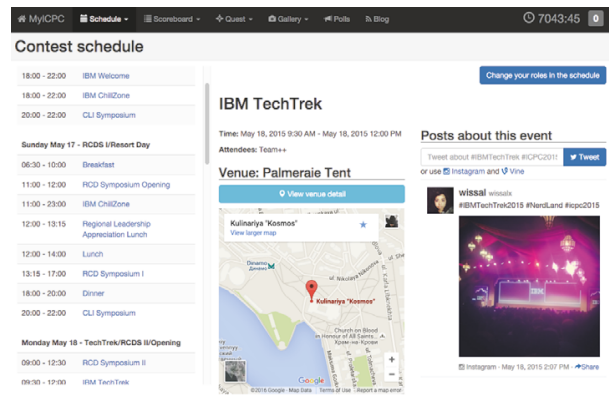

FIGURE 7: Schedule details page.

media, or even feedback from code analytics on source code progress (Figure 15). Live updates are displayed in multiple perspectives such as master scoreboard (Figure 12), compressed scorebar (Figure 16), world map (Figure 8), timeline (Figure 6), individual team perspectives, kiosk (Figure 18), etc. Figures 6-18 show selected perspectives of MyICPC.

In our study, we raise these questions to answer regards Second Screens:

(i) (Specialization) How interested is the local audience?

(ii) (Applicability) How applicable is the Second Screen to the global audience?

(iii) (Handle scale) What is the audience size?

(iv) How does the directed social gaming impact the social media events?

(v) Which device types are the most dominant and what are the technical characteristics of such devices, e.g., screen resolution and browser.

(vi) What is the SPEX classified feature popularity in a given time frame for the event?

(vii) How do feature interests differ between local and remote audience?

(viii) Are there any differences evaluating features by page views or visit time?

(ix) How does the device type influence the feature popularity?

At the time of publication, MyICPC was used four times at the World Finals and eight times in regional contests. In

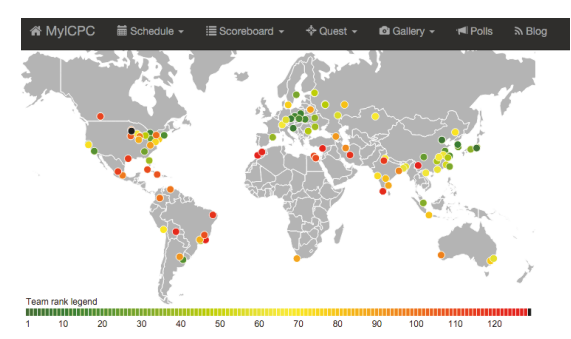

FIGURE 8: Interactive score world map.

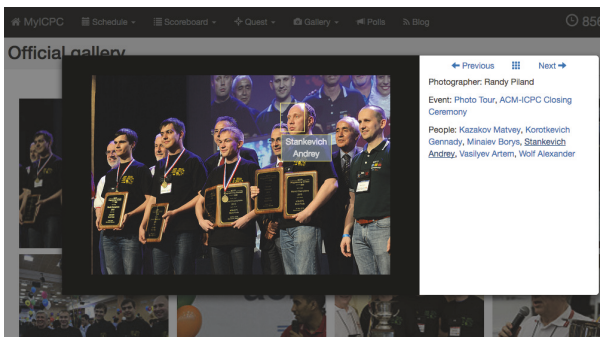

FIgURE 9: Media gallery with metadata.

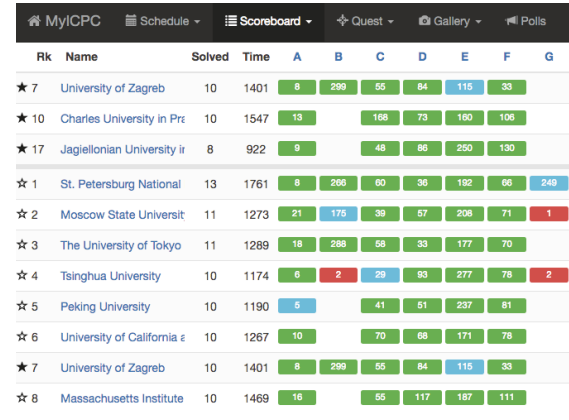

FIGURE 10: Scoreboard for desktop.

\begin{tabular}{|c|c|c|c|c|}
\hline \multicolumn{4}{|c|}{ 令 MylCPC } & 0 \\
\hline & 曲 & 湴 & 오 & : \\
\hline & Rk & Name & Solved & Time \\
\hline मे & 1 & St. Petersburg National Research Uni & 13 & 1761 \\
\hline मे & 2 & Moscow State University & 11 & 1273 \\
\hline मे & 3 & The University of Tokyo & 11 & 1289 \\
\hline मे & 4 & Tsinghua University & 10 & 1174 \\
\hline मे & 5 & Peking University & 10 & 1190 \\
\hline 它 & 6 & University of California at Berkeley & 10 & 1267 \\
\hline
\end{tabular}

FIGURE 11: Scoreboard for mobile/tablet.

the following sections, we consider access statistics harvested from the World Finals 2014-16. We consider audience access patterns for particular MyICPC perspectives to answer the above questions. The statistics involve MyICPC instances deployed for the 2014 Finals in Yekaterinburg, Russia; the 2015 Finals in Marrakesh, Morocco; and 2016 Finals in Phuket, 


\begin{tabular}{|c|c|c|c|c|c|c|c|c|c|c|c|}
\hline & My & iEScoreboard $-4 c$ & uest - & o Gallen & & Polls & ה Blog & & (). & 146 & $1 0 \longdiv { 2 }$ \\
\hline & Rk & Name & Region & Country & Solved & Time & A & B & c & D & E \\
\hline मे & 1 & Belarusian State Economic University & & BLR & 5 & 248 & & & & $1-66$ & $2-39$ \\
\hline 4 & 2 & Beijing Institute of Technology & & $\mathrm{CHN}$ & 3 & 138 & & & & 1-66 & \\
\hline मे & 3 & Saint Petersburg Academic University - Nanotechnol & & Rus & 3 & 196 & & $1-67$ & $1-66$ & $1-63$ & \\
\hline 4 & 4 & Indian Institute of Technology - Delhi & & IND & 2 & 86 & & & & & \\
\hline मे & 5 & St. Petersburg State University & & RUS & 2 & 125 & & & & $1-63$ & \\
\hline मे & 6 & Indian Institute of Technology - Kharagpur & & IND & 2 & 128 & & & & $1-65$ & \\
\hline th & 7 & University of Science and Technology of China & & $\mathrm{CHN}$ & 2 & 129 & & & & $1-65$ & \\
\hline मे & 8 & The University of Western Australia & & AUS & 2 & 130 & & & & $1-66$ & \\
\hline मे & 9 & Korea University & & KOR & 2 & 132 & & & $1-65$ & $1-67$ & \\
\hline 4 & 10 & Amrita School of Engineering, Kollam & & IND & 1 & 7 & 271 & & & & \\
\hline
\end{tabular}

FIGURE 12: Scoreboard real-time updates.

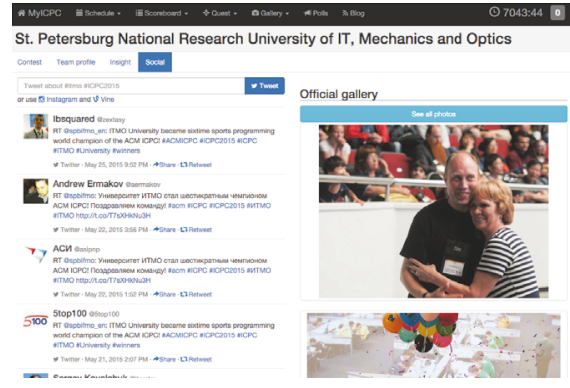

Figure 13: Team statistics and history.

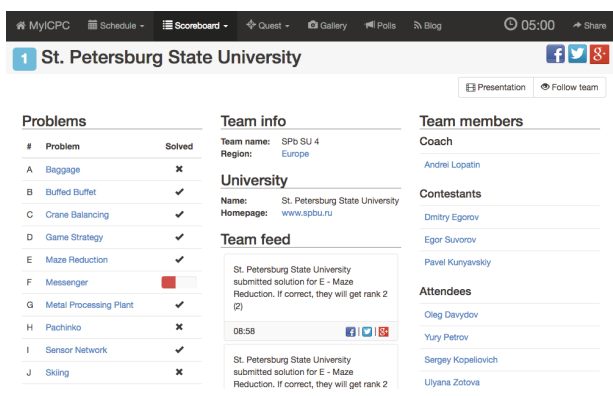

Figure 14: Team score statistics.

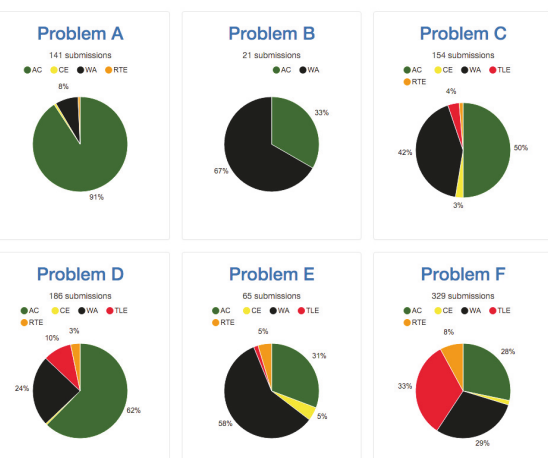

FIGURE 15: Code analytics

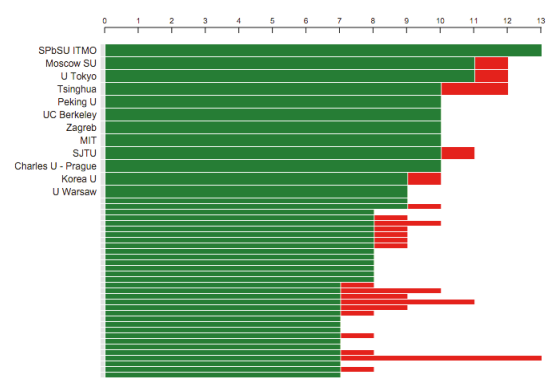

FIGURE 16: Scorebar.

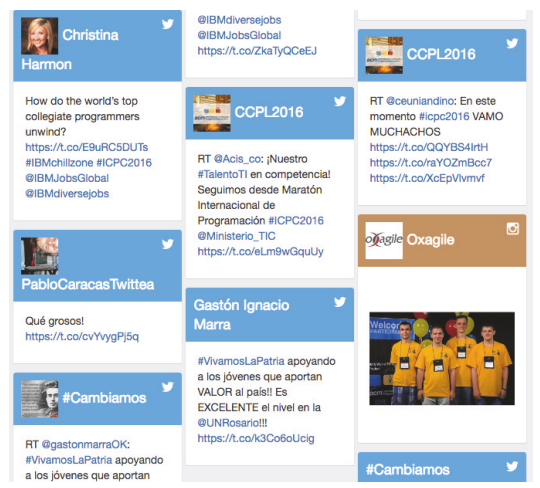

FIGURE 17: Kiosk content I.

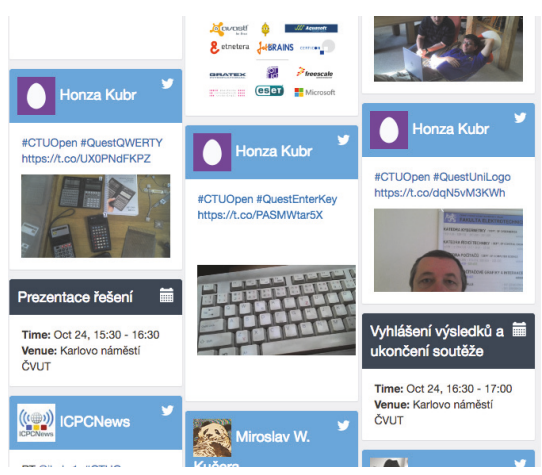

FIGURE 18: Kiosk content II. 
Thailand. For our evaluation, we consider the five days prior to the World Finals competition, the competition day itself and one day after for a total of seven days. For example, at the 2016 World Finals, we collected data starting May 14 and ending May 20. Collection for this period starts and ends at the 00:00 of the current local time zone (UCT+7). The statistics are gathered by Google Analytics [31], and Section 5.7 explains how the statistics are determined with details on the threats to validity.

5.1. Access Statistics. Table 1 gives a summary of the access data in multiple perspectives segments, such as the total audience in the considered period, contest day the audience, peak audience in an hour, average session time, number of sessions, and the total number of processed events.

The first segment local audience shows various audience perspectives across the years. The three lines show the registered attendees with the comparison to the audience visiting the organization website and MyICPC. While in the first year the MyICPC audience outnumbered the other monitored entries in the second and third years, it was around two hundred less than the number of registered attendees and $62-73 \%$ of the organization website audience.

The remote audience comparison between the MyICPC and organization website ranged from $37-48 \%$, which is less than the proportion for the local audience. The local audience is understandably lower than remote, although this is related to the local event attendees each year. Please note that multiple participants traveled throughout the considered period of time and thus they count to both remote and local numbers.

When considering the years, there are significant growths of the total audience for both entries. The total audience proportion considering MyICPC and the organization website gives $44 \%, 38 \%$, and $49 \%$ in years $2014-16$. This provides a good base estimate to carry out when one considers building a Second Screen for a particular community. Moreover, in our case, there are over four thousand new remote visitors each year and the proportion of local to registered audience remains almost constant, except 2014, which we discuss later in this section.

The contest day segment of Table 1 shows the contest day audience details. Considering the total audience number, the contest day itself makes the portion of $67-76 \%$ of the entire considered contest period. It is $68-77 \%$ portion for the remote audience and $48-54 \%$ portion for the local audience. Furthermore, notice that the contest day remote audience doubled over the considered years.

The important entry about scalability is the peak number of users per hour. It is important to know the scale to accommodate resources which will be utilized to serve the demands. From 2014 to 16 the peak audience in an hour grew up to 3'318 users per hour in the contest time. While the remote audience peak in an hour grew, the local audience peak reduced to half, although we consider a deviation in the 2014 local audience.

The statistics so far do not say much about the time spent on a page. For this evaluation, Google Analytics provides us with details on an average user session time and the total number of sessions per user. The local audience has longer, almost twice as long session. Moreover, the local audience has twice as many sessions than the remote audience. This behavior is related to the system features and content that are more focused on the local audience (Quest), e.g., sharing media.

Another considered criterion is the total number of processed events by the MyICPC, which considers the contest dates and include messages from Twitter, Instagram, Vine, administrator, etc. Next, we consider the impact of the social game, Quest, on social media, in particular on Twitter messages. Quest is the main motivation to be active on social media, in particular, Twitter, and targets the local audience, which targets the number of sessions per local user. The last segment in Table 1 shows the total number processed Twitter messages (with event hash) and also Quest-related messages (with both event and Quest hash). The statistics do not include Retweets messages or the long-term impact on social media. The number of Quest messages creates around $35-59 \%$ of the total Twitter messages. To depict the time effect, as of January 1, 2017, the Marrakesh Twitter with Retweets has grown to 16’329 messages and the Quest messages to 7’034.

From Table 1 we find answers to our questions above. Specifically, it shows the local MyICPC audience in the contrast to the organization website and the registered attendees, from which we can derive the portion of the local audience engage with MyICPC (62-100\% of the organization website audience). The remote audience is significantly larger, even though, the local audience spends more time on the MyICPC. In 2016 there was an audience of 1'307 users each with an average of 3.11 sessions lasting on average 15 minutes and 10 sec. This indicates that each local user spent over 47 minutes on the MyICPC. In 2015 it was almost an hour. The audience ratio ranges from 5.6 to 15.8 in favor of the remote audience. This answers Questions (i) and (ii).

The important observation is the peak number of visitors in a particular time frame. The peak number of visitors makes the system administer the critical information on system resource demands. In our case, 3'218 users visited the system during the peak hour. The peak day had the load of sixteen thousand unique visitors. Considering the program across the years in Table 1 we may expect up to twenty thousand visitors in the peak day and around three and half thousand users in the peak hour. This answers our Question (iii).

At this point, we might be interested in the audience daily load. Figure 19 shows the remote and local audience load in a given day, relative to the contest, plotted on a logarithmic scale. Moreover, we see all the considered years 2014-2016 that have similar characteristics. We can note the different progression for the remote audience that peaks around the contest day, while the local audience load spreads across the days.

Considering the total events processed; approximately $35-$ $59 \%$ of Twitter messages are Quest-related messages, which shows Quest's significant impact on social media, in particular, Twitter. Moreover, Quest is played by the local audience, and thus around $50 \%$ of social media produced from the event in 2016 is stimulated by Quest, which is remarkably high percentage and answers Question (iv). 
TABLE 1: World Finals 2014-16 statistics.

\begin{tabular}{|c|c|c|c|}
\hline & $\begin{array}{c}\text { Yekaterinburg } \\
2014 \\
\end{array}$ & $\begin{array}{c}\text { Marrakesh } \\
2015 \\
\end{array}$ & $\begin{array}{c}\text { Phuket } \\
2016 \\
\end{array}$ \\
\hline \multicolumn{4}{|l|}{ Local audience } \\
\hline Local registered attendees & $1 ' 227$ & $1^{\prime} 170$ & $1 ' 544$ \\
\hline Local audience at organization website & 2’006 & 1’586 & $1 ' 780$ \\
\hline Local audience MyICPC & $2^{\prime} 027$ & 977 & 1'307 \\
\hline \multicolumn{4}{|l|}{ Remote audience } \\
\hline Remote audience at organization website & $288^{\prime} 230$ & $41^{\prime} 447$ & $411^{\prime} 593$ \\
\hline Remote audience MyICPC & $11 ' 288$ & $15^{\prime} 500$ & $20 ’ 095$ \\
\hline \multicolumn{4}{|l|}{ Total audience } \\
\hline Total audience at organization website & $29 ’ 991$ & $42^{\prime} 718$ & $43^{\prime} 043$ \\
\hline Total audience MyICPC & $13^{\prime} 153$ & $16^{\prime} 333$ & $21 ’ 204$ \\
\hline \multicolumn{4}{|l|}{ Contest day } \\
\hline Contest day local audience & 1’092 & 472 & 711 \\
\hline Contest day remote audience & $7^{\prime} 748$ & $11^{\prime} 640$ & $15 ’ 419$ \\
\hline Contest day total audience & $8^{\prime} 814$ & $12 ’ 099$ & $16^{\prime} 019$ \\
\hline \multicolumn{4}{|l|}{ Peak in hour } \\
\hline Max local visitors in one hour & 230 & 140 & 115 \\
\hline Max remote visitors in one hour & $1 ’ 576$ & $2{ }^{\prime} 816$ & 3’117 \\
\hline Max total visitors in one hour & 1'806 & 2'929 & 3’218 \\
\hline \multicolumn{4}{|l|}{ Average session } \\
\hline Average user session - local [mm:ss] & $11: 25$ & $15: 38$ & 15:10 \\
\hline Average user session - remote [mm:ss] & $7: 02$ & $10: 21$ & $7: 45$ \\
\hline Average user session - total [mm:ss] & $8: 11$ & 10:59 & $8: 33$ \\
\hline \multicolumn{4}{|l|}{ Number of session } \\
\hline Sessions per user - local & 3.35 & 3.72 & 3.11 \\
\hline Sessions per user - remote & 1.69 & 1.72 & 1.69 \\
\hline Sessions per user - total & 1.96 & 1.86 & 1.79 \\
\hline \multicolumn{4}{|l|}{ Total events } \\
\hline Events processed & $5 ’ 615$ & 4’305 & 7’057 \\
\hline Twitter messages (excludes Rerweets) & 4'290 & 3’876 & 6539 \\
\hline Quest messages (excludes Retweets) & 1'491 & $2^{\prime} 057$ & 3’832 \\
\hline
\end{tabular}

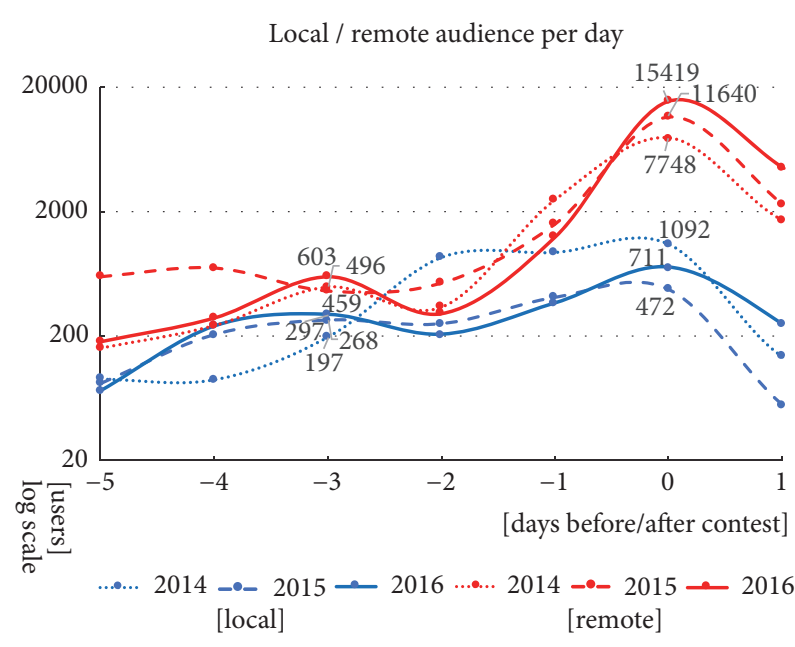

FIGURE 19: Daily remote/local audience load for the 2014-2016 year.
Next, we aim to answer Question (v) and show the characteristics of the particular audience group. Tables 2-4 show details on the remote and local audience details and proportion of devices, characteristics, operating systems, and browsers. In particular, we can see that the dominant device type is a desktop for the remote audience, although they use a rather high percentage of mobile phones and this trend has grown over the years. This trend must be considered in the user interface design of future systems. On the other hand, the local the audience tends to limit the tablet use and the percentage of phones and desktops is similar in favor of desktops the last year.

Similarly, Windows is the dominant platform; however, Android almost doubled between 2014 and 2016 for the remote audience. For the locals, iOS lost dominance, while Windows gained the popularity. The pallet of screen resolutions is dominated by $1366 \times 768$ for the remote, and $360 \times 640$ 
TABLE 2: World Finals 2014-16 total audience details (TOTAL).

\begin{tabular}{|c|c|c|c|c|}
\hline & Yekaterinburg (2014) & Marrakesh (2015) & \multicolumn{2}{|c|}{ Phuket (2016) } \\
\hline Desktops & $75 \%$ & $74 \%$ & $70 \%$ & \\
\hline Mobiles & $20 \%$ & $23 \%$ & $27 \%$ & \\
\hline Tablets & $5 \%$ & $3 \%$ & $3 \%$ & \\
\hline $1^{\text {st }}$ operating system & Windows $\quad 54 \%$ & Windows $\quad 50 \%$ & Windows & $48 \%$ \\
\hline $2^{\text {nd }}$ operating system & Android $\quad 13 \%$ & Android $\quad 17 \%$ & Android & $21 \%$ \\
\hline $3^{r d}$ operating system & Mac $\quad 11 \%$ & Mac $\quad 13 \%$ & Mac & $12 \%$ \\
\hline $4^{\text {th }}$ operating system & iOS/Linux $\quad 11 \%$ & Linux & Linux & $11 \%$ \\
\hline $1^{\text {st }}$ web browser & Chrome $\quad 60 \%$ & Chrome & Chrome & $67 \%$ \\
\hline $2^{\text {nd }}$ web browser & Firefox $\quad 16 \%$ & Firefox $\quad 15 \%$ & Firefox & $13 \%$ \\
\hline $3^{r d}$ web browser & Safari $\quad 13 \%$ & Safari & Safari & $10 \%$ \\
\hline $1^{\text {st }}$ screen resolution & $1366 \times 768 \quad 32 \%$ & $1366 \times 768 \quad 31 \%$ & $1366 \times 768$ & $30 \%$ \\
\hline $2^{\text {nd }}$ screen resolution & $1920 \times 1080 \quad 11 \%$ & $1920 \times 1080 \quad 13 \%$ & $1920 \times 1080$ & $14 \%$ \\
\hline $3^{\text {rd }}$ screen resolution & $1440 \times 900 \quad 7 \%$ & 1440x $900 \quad 7 \%$ & $360 \times 640$ & $12 \%$ \\
\hline
\end{tabular}

TABLE 3: World Finals 2014-16 total audience details (REMOTE).

\begin{tabular}{|c|c|c|c|c|}
\hline & Yekaterinburg (2014) & Marrakesh (2015) & \multicolumn{2}{|c|}{ Phuket (2016) } \\
\hline Desktops & $81 \%$ & $76 \%$ & $71 \%$ & \\
\hline Mobiles & $16 \%$ & $21 \%$ & $26 \%$ & \\
\hline Tablets & $3 \%$ & $3 \%$ & $3 \%$ & \\
\hline $1^{\text {st }}$ operating system & Windows $\quad 59 \%$ & Windows $51 \%$ & Windows & $49 \%$ \\
\hline $2^{\text {nd }}$ operating system & Linux $\quad 12 \%$ & Android $\quad 16 \%$ & Android & $21 \%$ \\
\hline $3^{\text {rd }}$ operating system & Mac $11 \%$ & Mac $12 \%$ & Mac & $12 \%$ \\
\hline $4^{\text {th }}$ operating system & Android $\quad 11 \%$ & Linux $\quad 12 \%$ & Linux & $11 \%$ \\
\hline $1^{s t}$ web browser & Chrome $\quad 64 \%$ & Chrome & Chrome & $68 \%$ \\
\hline $2^{\text {nd }}$ web browser & Firefox $\quad 17 \%$ & Firefox $\quad 15 \%$ & Firefox & $14 \%$ \\
\hline $3^{\text {rd }}$ web browser & Safari $\quad 9 \%$ & Safari $10 \%$ & Safari & $9 \%$ \\
\hline $1^{\text {st }}$ screen resolution & $1366 \times 768 \quad 35 \%$ & $1366 \times 768 \quad 31 \%$ & $1366 \times 768$ & $31 \%$ \\
\hline $2^{\text {nd }}$ screen resolution & $1920 \times 1080 \quad 12 \%$ & $1920 \times 1080 \quad 14 \%$ & $1920 \times 1080$ & $14 \%$ \\
\hline $3^{\text {rd }}$ screen resolution & $1440 \times 900 \quad 7 \%$ & $1440 \times 900 \quad 7 \%$ & $360 \times 640$ & $12 \%$ \\
\hline
\end{tabular}

TABLE 4: World Finals 2014-16 local audience details (LOCAL).

\begin{tabular}{|c|c|c|c|}
\hline & Yekaterinburg (2014) & Marrakesh (2015) & Phuket (2016) \\
\hline Desktops & $763 \quad 38 \%$ & $49 \%$ & $50.5 \%$ \\
\hline Mobiles & $958 \quad 48 \%$ & $42 \%$ & $43.5 \%$ \\
\hline Tablets & $275 \quad 14 \%$ & $9 \%$ & $6 \%$ \\
\hline $1^{\text {st }}$ operating system & iOS $33 \%$ & Android $28 \%$ & Windows $29 \%$ \\
\hline $2^{\text {nd }}$ operating system & Android $\quad 27 \%$ & iOS $\quad 23 \%$ & Android $\quad 27 \%$ \\
\hline $3^{r d}$ operating system & Windows $\quad 20 \%$ & Windows $\quad 22 \%$ & iOS $\quad 22 \%$ \\
\hline $1^{\text {st }}$ web browser & Chrome $37 \%$ & Chrome $56 \%$ & Chrome $\quad 60 \%$ \\
\hline $2^{\text {nd }}$ web browser & Safari $\quad 35 \%$ & Safari $\quad 24 \%$ & Safari $\quad 25 \%$ \\
\hline $3^{r d}$ web browser & Firefox $\quad 10 \%$ & Firefox $\quad 13 \%$ & Firefox $9 \%$ \\
\hline $1^{\text {st }}$ screen resolution & $320 \times 568 \quad 12 \%$ & $1366 \times 768 \quad 17 \%$ & $360 \times 640$ \\
\hline $2^{\text {nd }}$ screen resolution & $1366 \times 768 \quad 12 \%$ & $360 \times 640 \quad 16 \%$ & $1366 \times 768$ \\
\hline $3^{\text {rd }}$ screen resolution & $320 \times 480 \quad 11 \%$ & $1280 \times 800 \quad 7 \%$ & $1920 \times 1080 \quad 9 \%$ \\
\hline
\end{tabular}


for the locals. This requires the responsive user interface design considering a broad range of resolutions, with the content visible and attractive.

Specifically, the local audience that we measure and summarize in Table 4 tends to be more mobile-oriented, but still, in the last two years, desktops lead usage. There is a significant competition among Windows, Android, and iOS over the year. This shows that building a single operating-systemoriented the application does not address the wide-range of the audience. While the Chrome browser is still the dominant browser, a significant portion of the audience uses Safari and thus web application must be compatible across a wider range of web browsers. In future we expect the screen resolution to get higher with the trends of modern mobile phones.

When we return back to Table 1, we already mentioned the annual growth in the remote audience but also note the reduction of the local audience in 2015 . What could be the reason behind this? Unfortunately, there is no simple answer. Thus, we provide some conjecture points. The 2014 event took place in Russia, and the Russian teams are repeat champions, which may have played a role in increased interest. Next, the Yekaterinburg venue took place in a public stadium in the city center with mass media and local host university support, while Marrakesh was in the private Palmeraie Resort, distant from the center of Marrakesh. In 2016 the physical deployment was similar to 2014. Another possible explanation could be that in 2014 the host provided all attendees with a prepaid data plan SIM-card. This allowed them to use their phone and access Internet in any public location in Russia. The usage of mobile phones almost doubles in 2014 over the other years, and this could play a role in the attendee involvement. Specifically, for the 2014 event, Google Analytics identifies 145 local visitors accessing through GPRS service provider, but only 2

\section{Scoreboard}

Presents real-time actual score during the contest or practice contest, relates to the main event. Allows highlighting particular teams and following their updates.

\section{Quest}

Social media game involving solving tasks for the local contest participants.

\section{Team statistics and history}

Details on teams, members, integrate social media, photographs, submissions or code analysis.

\section{Timeline}

Integrates all events and notifications to a time-ordered, self-updating timeline. Highlights events from schedule and supports interaction by prefiled links to each notification.

\section{Live stream}

Live video broadcast.

\section{Code analytics}

Related to the contest/practice providing insight information on teams source code analysis.

\section{Scorebar}

Graphical compressed version of scoreboard fits to screen.

\section{Interactive map}

Graphical highlight of score on World map or Regional map.

\section{Problem view}

Highlights global view on code changes in last 5 minutes, submitted problems and their accept/reject ratio with specific submission details. in 2015 and 42 in 2016. In 2014, 123 mobile network visitors out of the total 145 used the SIM provider distributed by the host.

5.2. SPEX Model Classification of MyICPC Features. The SPEX model provides a good base for features to support interaction. In this section, we classify the MyICPC features according to the model.

The MyICPC supports social involvement for the local audience with Quest (Figures 4 and 5) and timeline (Figure 6). The audience can direct social messaging to particular events (Figure 10), teams (Figure 13), or schedule (Figure 7) using hash-tags. Their posts are distributed to particular pages based on these hash-tags. The media shared with the (Figure 9). The MyICPC (Figure 14) and Next, the schedule is provided with its program, views on particular team submissions (Figproviding teams pictures, team history, etc.

We select the most popular pages by the access statistics, elaborated in the later section, and classify them by SPEX. For each considered page, we highlight the aspect of the discipline $[D]$, event $[E]$, or social involvement $[S]$ which matches the SPEX model. Moreover, we annotate the page's option to provide a personalized view $[P]$.

$$
[E|D| S \mid P]
$$

$[E|D| S \mid P]$

$[E|D| S]$

$[E|D| S]$ 


\section{Schedule}

Event program with links to venue, map allowing personalizing the schedule.

5.3. Audience Temporal Interest Classification. We consider two audience dimensions, the local versus remote audience and their interaction in a certain time frame. Certain functionality boosts the interests and user involvement before, during, and after the main event, the actual contest.

For instance, Quest (Figures 4 and 5) supports the local audience interaction before the contest and so does the timeline (Figure 6). Since audience can direct social messaging to particular events (Figure 10), teams (Figure 13), or schedule (Figure 7), they have a certain control over the content. At the same time, administrators can restrict the posts.

Certain features impact the local / remote audience before the event, during the main event, and after it. In order to evaluate the audience interest and classify it by the time frame and location, we select the most popular pages and process them by the access statistics.

In particular, there are two possible statistics to consider. The total page hits or the page visit time. The page hits fit well to static pages. Since the MyICPC pages are interactive, using push technology, they allow the user to follow automated page updates with no page redirects or reloads. For this reason, we focus on the statistics involving page visit time. This considers the time spent on a page until a page redirects or reload happens. The details of the evaluation are provided in the next section.

5.4. Statistics on Feature in Terms of Time at Page. Next, we draw the audience interest, in particular, MyICPC features on given pages. These statistics consider the local and remote audience during a slightly different period that considered earlier. The 2015 Finals are considered. The period considered in this section divides into 120 hours before the contest, 10 hours during the contest ( 3 hours before and 2 hours after it counted to this fragment), and 38 hours after the contest. Table 5 presents the main highlights and the following text provides details.

The local audience spent almost one thousand hours on MyICPC, 67\% before the main event, 28\% during the main event, and only 5\% after. Quest is the dominant feature with $45 \%$ of the audience time and even $62 \%$ of the time before the event. The scoreboard is the most dominant during the event, but overall it is high as the second most popular feature with $24 \%$ of the audience attention. The popularity of the scoreboard during the main event is a remarkable $62 \%$. The team statistics and history, timeline, and schedule receive significant local audience interest. While the team statistics and history keep the popularity throughout the considered time frames, the timeline loses the audience during the main event. Moreover schedule is used mostly before the main event. Other pages give only marginal interest to the local audience.

In comparison, the remote audience shows significant differences. In total, the audience spent 4'601 hours on MyICPC with the division onto $10 \%$ before, $70 \%$ during, and $20 \%$ after the main event. Most importantly $74 \%$ of the time, the audience followed the scoreboard. This correlates to the local audience interest and the characteristics from Figure 19. The most obvious is the lack of interest in Quest, which has the goal and purpose to get local audience attention. Even though Quest gets higher attention before the event, the overall drops to marginal. A long gap after the scoreboard is followed by the team statistics and history with rather constant interest $12 \%$. The timeline gets the remote audience attention mostly before the main event and settles on $4 \%$ during the main event. Other features, such as code analytics, schedule, or live stream receives only marginal interest. The statistics show rather conservative interests of the remote audience.

From the SPEX model perspective, Quest relates to the event and mostly to the social aspect of the local audience. Scoreboard is related to the event and discipline; it supports the social aspect, but much less than Quest. The timeline, which is similar to Quest, is the central location for the social interaction; moreover, the content is related to the event and the discipline. For the team statistics and history, the discipline is the dominant aspect; the event and social aspects are rather secondary. Schedule is related to the event aspect, and its marginal part is the social involvement. This gives the answer to Question (vi).

Furthermore, we must highlight that the SPEX model does not target the remote audience. Additionally, Table 5 shows the differences in the interests in features for the remote and local audience and answers the Question (vii).

Finally, we answer Question (viii) considering Table 5. From the page visits statistics, scoreboard has shown only $13 \%$ interest of the local audience and $48 \%$ for the remote audience. The page visit time shows a significantly higher proportion. The reason behind this is that users load it once and follow the page push updates. On the contrary, team statistics and history indicate high interest for the remote audience by page visits, reaching $24 \%$, but the time on the page is half, similarly to timeline with page visits $9 \%$ remote audience interest in the pages, but with the time one page only a half. Both statistics can give specific perspective, although the time on page fits better to the interactive applications using push communication. For completeness, we show the page visit statistics in Table 6 .

5.5. Event Statistics by Time on Page and Device. In this section, we consider the contest statistics and page popularity with respect to time on page by a particular device type to answer the Question (ix). These statistics involve the 2015 World Finals. We consider the time of five hours during the contest for both local and remote audience. Next, we also consider the 120 hours before the event for the local audience. The summary and overview are provided by Table 7 .

The main event has similar distribution of device types for the local audience. The top feature is scoreboard. The desktop users show higher interest in timeline and teams statistics and even live stream; on the other hand, they had less interest in scoreboard than others. Even though the mobile phones 
TABle 5: Page visit time statistics [\%].

\begin{tabular}{|c|c|c|c|c|c|c|c|c|}
\hline \multirow[b]{2}{*}{ Feature } & \multicolumn{4}{|c|}{ Local audience } & \multicolumn{4}{|c|}{ Remote audience } \\
\hline & Total & Before & During & After & Total & Before & During & After \\
\hline Hourly distribution [h] & 948 & 632 & 268 & 47 & 4'601 & 466 & 3’219 & 916 \\
\hline Quest & 45 & 62 & 8 & 34 & 1 & 23 & $<0.5$ & 2 \\
\hline Scoreboard $_{\text {[practice] }}$ & 24 & 7 & 62 & 36 & 74 & 27 & 75 & 73 \\
\hline Timeline & 10 & 10 & 2 & 10 & 4 & 17 & 4 & 5 \\
\hline Team stat. \& history & 9 & 9 & 12 & 13 & 12 & 16 & 11 & 12 \\
\hline Schedule & 6 & 7 & 2 & 3 & 1 & 5 & 1 & 1 \\
\hline Live stream & 1 & $<0.5$ & 2 & $<0.5$ & 1 & 3 & 1 & $<0.5$ \\
\hline Scorebar & 1 & $<0.5$ & 1 & 2 & 1 & $<0.5$ & 1 & 1 \\
\hline Code analytics & 1 & 1 & 2 & $<0.5$ & 2 & 2 & 2 & 1 \\
\hline
\end{tabular}

TABle 6: Page view statistics [\%].

\begin{tabular}{|c|c|c|c|c|c|c|c|c|}
\hline \multirow[b]{2}{*}{ Feature } & \multicolumn{4}{|c|}{ Local audience } & \multicolumn{4}{|c|}{ Remote audience } \\
\hline & Total & Before & During & After & Total & Before & During & After \\
\hline Visits distribution & - & 79 & 16 & 5 & - & 18 & 56 & 26 \\
\hline Quest & 53 & 62 & 13 & 30 & 5 & 17 & 2 & 4 \\
\hline Scoreboard ${ }_{\text {[practice] }}$ & 13 & 6 & 42 & 25 & 48 & 21 & 54 & 50 \\
\hline Team stat. \& history & 11 & 10 & 17 & 20 & 24 & 31 & 22 & 23 \\
\hline Timeline & 10 & 9 & 12 & 12 & 9 & 15 & 6 & 9 \\
\hline Schedule & 6 & 9 & 1 & 1 & 2 & 2 & 1 & 2 \\
\hline Problem view & $<0.5$ & $<0.5$ & $<0.5$ & $<0.5$ & 4 & $<0.5$ & 4 & 5 \\
\hline
\end{tabular}

TABLE 7: Time on page by device, time fragment, and audience.

\begin{tabular}{|c|c|c|c|c|c|c|c|c|c|}
\hline \multirow{3}{*}{$\begin{array}{l}\text { Event frame } \\
\text { Audience } \\
\text { Device } \\
\end{array}$} & \multicolumn{6}{|c|}{5 hours during event } & \multicolumn{3}{|c|}{ 120h before the event } \\
\hline & \multicolumn{3}{|c|}{ Local } & \multicolumn{3}{|c|}{ Remote } & \multicolumn{3}{|c|}{ Local } \\
\hline & Mobile & Tablet & Desktop & Mobile & Tablet & Desktop & Mobile & Tablet & Desktop \\
\hline Total audience[-] & 122 & 35 & 114 & 1'219 & 174 & 4775 & 298 & 61 & 381 \\
\hline \multicolumn{10}{|l|}{ Page/feature [\%] } \\
\hline Scoreboard & 73 & 70 & 60 & 77 & 81 & 74 & 6 & 10 & 9 \\
\hline Quest & 8 & 7 & 4 & $<0.5$ & $<0.5$ & $<0.5$ & 65 & 70 & 57 \\
\hline Timeline & 6 & 8 & 10 & 6 & 7 & 4 & 9 & 4 & 12 \\
\hline Teams stat. & 6 & 11 & 12 & 7 & 7 & 12 & 10 & 3 & 8 \\
\hline Schedule & $<0.5$ & 2 & 2 & $<0.5$ & $<0.5$ & $<0.5$ & 7 & 8 & 7 \\
\hline Live stream & $<0.5$ & 2 & 4 & 2 & $<0.5$ & 1 & $<0.5$ & $<0.5$ & $<0.5$ \\
\hline Scorebar & 2 & $<0.5$ & $<0.5$ & 2 & $<0.5$ & 1 & $<0.5$ & $<0.5$ & $<0.5$ \\
\hline Code analytics & 2 & $<0.5$ & $<0.5$ & $<0.5$ & $<0.5$ & 2 & $<0.5$ & $<0.5$ & 2 \\
\hline
\end{tabular}

have limited screen resolution; they followed the scoreboard the most. Interestingly enough, the local audience had interest in Quest, while the remote had almost none.

The most dominant device type for the remote audience is desktop, with almost four times as many devices than mobiles. Tablet usage is marginal. There is the same interest in the main feature scoreboard. The desktop local audience users more followed the team statistics and history. On the other hand, desktop users followed much less the timeline than the local audience. We can see different interest in Quest during the main event between both audiences.
For the local audience before the contest, there is a similar distribution for mobiles and desktops and few tablet users. Naturally, the screen size differs as well as the ability to interact. Mobile and tablet users were more active in Quest. This is mostly because of Twitter app integration, which uses the embedded camera for messaging. The desktop users followed the timeline more than others. Tablet users were most active in Quest and followed the timeline and team statistics and history much less than others.

Both statistics during the main event show the importance of the scoreboard; other features and pages are only 


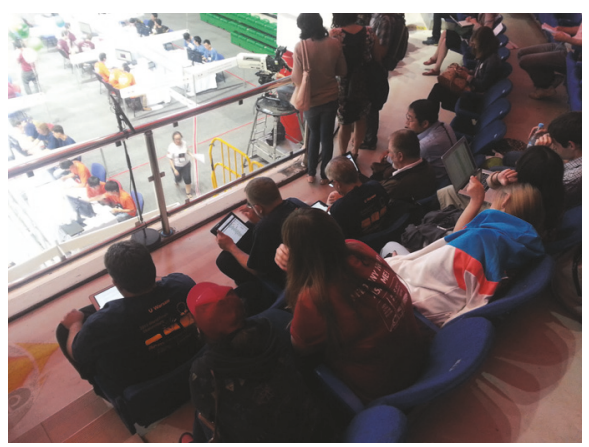

Figure 20: Local audience using MyICPC.

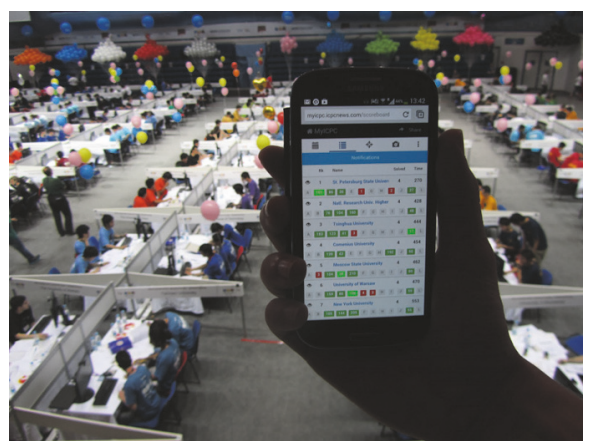

FIGURE 21: Real-time scoreboard in action.

secondary when considering the main event. It seems beneficial to provide the team statistics and history and the timeline and for the local audience additional entertainments such as Quest, but other functionalities raise marginal interest to the audience.

To compare the interests for the local audience in different time frames, Table 7 provides both the main event and before event perspectives. We may notice the flip from Quest and scoreboard during the main event as well as increased interest for the schedule. Figure 20 shows the local audience using MyICPC at the World Finals. Figure 21 shows the real-time progress on scoreboard on a mobile phone during the contest.

5.6. Discussion of Study Results. Our aim in the study was to demonstrate a real production system that reflects our architecture and design suggestions and evaluates the audience interests and characteristics. The system considers many features based on the SPEX model. The evaluation should give the reader practical feedback on audience properties and interest. In the study, we consider both remote and local audience, since their interest differs. We consider their devices, screen sizes, operating systems, and even web browsers showing that the Second Screen should expect quite heterogeneous client devices and platforms. Thus, building a native application, e.g., an Android app, would reduce the audience scope only to approximately $20-30 \%$. Next, the design investment should consider various screens ranging from 320x480 to 1920x1080, while future systems may expect to increase the lower bound resolution range. In case of web applications, they must be optimized for various web browsers.

Specifically, when addressing local audience, we expected that the target device is a portable device such as mobile phone or tablet, but our evaluation shows significant use of desktops. This might be influenced by the domain since it is hard to imagine spectator with notebooks in football match. To boost and mediate the participant interaction a social gaming preceding the main event seems like a great instrument. In our case, Quest is the source of almost 50\% of all processed events. We should point out that these events do not only impact the Second Screen but also all participants social network allowing distant friends to share the individual's experience. The evaluation of the rest of considered system features shows that the audience mostly uses core features, in particular scoreboard and team statistics and history. The local audience shows demands for event-related information such as schedule and timeline.

Based on the timing, we can expect that remote audience waits for the main scoreboard and in the meantime monitors the local audience activity on the Second Screen, but the peak audience arrives for the main event. Local audience shows a nice transition from the social gaming interests before the event into the main event interest once the right time comes. Naturally, from the local audience perspective there are more demands on the variety of features. Finally, many of provided features consumed a lot of time and investment to develop but receive only a marginal interest from the audience.

There are not really significant interest variations for users with particular devices. Even the mobile phone users access mostly the scoreboard no matter the screen size. There might be additional support for particular devices from third parties, such as Twitter app integrating phone camera and the Second Screen should take advantage of them. There is perhaps one interesting deviation in the local audience with desktops and it is the interest in the live stream video. This might be related to users sitting in less convenient locations or hallways following the show privately.

5.7. Threats to Validity. Next, we discuss the threats to validity regarding the study result outcome as well as the possible generalization of results. We divide the text into internal and external validity.

Internal validity: the source of information that we base our evaluation on is a production-level service, Google Analytics, used in industry and research [32]. Unfortunately, this does not guarantee the correctness of the statistical results [31]. We use the free version of the services that has limitations. The paper [31] shows limitations that are related to the total hit limit per month $10 \mathrm{M}$ (page view/event), table aggregation, 500 hits per session, limited event tracking, and revenue strategies, etc. Fortunately, we do not cross the limits.

For Google Analytics, each page loaded by the end user contains a small JavaScript fragment that contacts Google Analytics service indicating the application key accompanied by HTML5 statistics on performance and context. Google Analytics identifies users through the client ID field, a unique, randomly generated string that gets stored in the browser's cookies. This allows Google Analytics to identify users across 
multiple sessions, even when IP address changes, but not across multiple browsers or devices.

The paper [31] admits Google Analytics inaccuracies. For instance, client-side caching or web accelerators can cache the pages and thus not count repeated visits. Robots and webcrawlers are counted, and over a long sampling interval, this over-counting can be drastic, although our interval was short. Pages with JavaScript errors halt the processing and thus will not show in the statistics, although this is not our case. Certain users with disabled JavaScript (e.g., 2.6\% in US based on [31]) will be not counted. Certain firewalls can remove some tags or cookies. In such cases, only the IP address is counted, but [31] states that users can dynamically receive different IP addressed from their Internet service Provider or due to relocation. Users can delete cookies and thus impact the statistics. The paper [31] emphasizes that measuring unique web visitors is fraught with many problems, mostly relevant to longer periods of days and to lost/blocked/removed cookies. Fortunately, our sampling period was not long. Another significant issue is browsing in the incognito mode that skews the statistics, as cookies are not kept across browser sessions. The unique user calculation issue is mostly related to statistics for users with multiple devices or users sharing devices.

A study evaluating accuracy of the Google Analytics performed in (http://www.blastam.com/blog/index.php/2013/02/ can-you-trust-your-google-analytics-data) suggests that visits statistics show variation in accuracy $0.89-1.46 \%$. The results are much worse for transactions, revenue, or e-commerce conversion rate, which we did not consider in the study. While not considering full accuracy on the statistics from Google Analytics, it gives us a reasonable approximate feedback on the audience. The classification to Mobile/ Table/Desktop users gives us a detailed statistic on user devices, operating systems, or flash versions.

We also need to emphasize the way Google Analytics determines time on page, which is perhaps the biggest threat to validity. It considers page transitions, where the transition to a next page determines time spent on the current page. On the last page, there is no next page so the time on page ends when the user opens the last page, which skews the statistics. In the extreme case user only visits a single page, the page visit count is 1 but the time on page is 0 ! In our case if the last page was interactive, such as timeline, scoreboard, scorebar, map, and problem view, the time on page is counted 0 ; thus, our statistics reports a lower bound with respect to time. At the same time, the total number of visitors can be lower.

External validity: our application is representative of a real-world application. The features provided by the application reflect the suggestions given by related work and their base reflects SPEX model. The example is specific to programming contests, although other contexts for the event such as conferences, sports matches, or exhibitions are expected to provide similar features and deal with similar issues or audience distribution. We cannot say that our representation reflects all aspects of future Second Screens. Our example demonstrates practical use and production experience with a Second Screen application. The locations of World Finals represent two particular scenarios providing real-world results. We believe that many noncomputing aspects play a role in the usage by the audience, such as cultural and economic factors or Internet access. The study considered computersavvy users, as we believe that top programmers and IT staff (participants of the evaluated events) more likely use similar application.

\section{Conclusion}

In this article, we analyze factors impacting audience involvement and interest in using a Second Screen application. We consider a wide spectrum of audiences, such as home users and active audience using a mobile device as well as regular attendees of the events.

We suggest what to consider when performing an analysis of a Second Screen application that can be accessed by users through conventional web browsers. These users can use their mobile or even desktop devices while receiving real-time services adapting to various contexts, interests, or screens serving both the local and remote audience.

We share a case study involving a Second Screen application called MyICPC. The study involves a large event, the World Finals in Programming. We described particular details on available features and evaluate audience interest considering different time frames, local versus remote audience, or given devices. Out of the statistics provided by Google Analytics, we believe that the majority of the local audience used MyICPC to augment their live experience. We demonstrate audience interest in certain features. In particular, we emphasize interest of the local audience that spent the most time with the social game Quest with peak interest before the event. The scoreboard received the highest remote audience interest during the main event. The remote audience seems to be more conservative than the local. While there are differences in certain interests among the audience such as the schedule page usage, the case study shows that the main design interest should be placed to features such as the scoreboard and social gaming before the event, the timeline, the team statistics, and historical data, and to the schedule. Other features received unimportant audience interest.

Second Screen applications seem to be an evolutionary step to augment large events and venues. Nowadays spectators equipped with ever-improving mobile devices aim to receive the best perspectives, information from the event, or augmenting information. All these services provided through Second Screen increase the quality of their experience. Second Screen applications can impact new audiences and motivate passive users to contribute to their personal experience to the crowd media. Furthermore, this article highlights the benefits, potential, and motivation elements for such applications. While having the production-level experience, we share our design decisions so that reader may avoid prototyping issues. Next, in a particular context, we demonstrate the application of discussed features that support the social/event and discipline aspects of the audience. Finally, we show the real-world audience interest distribution to considered pages with described functionality.

This research contributes to the area of Second Screen applications and their utilization for various events to impact individuals from various angles, providing a personalized 
form of experience. From the future perspective, there is a high potential for utilization for learning purposes. For instance, it can improve educational settings and be used during conferences or even in the classroom when multiple channels can augment the mainstream of information for the audience.

\section{Data Availability}

The data used to support the findings of this study are available from the corresponding author upon request.

\section{Disclosure}

This manuscript is an extended version of a paper presented at 2016 6th International Conference on IT Convergence and Security (ICITCS) [33].

\section{Conflicts of Interest}

The authors declare that they have no conflicts of interest.

\section{Acknowledgments}

Our research was supported by the ACM-ICPC/Cisco Grant 0374340 .

\section{References}

[1] N. Staelens, S. Moens, W. Van Den Broeck et al., "Assessing quality of experience of IPTV and video on demand services in real-life environments," IEEE Transactions on, vol. 56, no. 4, pp. 458-466, 2010.

[2] K. Hyun-Wook, R. M. Hoque, S. Hyungyu, and S. H. Yang, "Development of middleware architecture to realize contextaware service in smart home environment," Computer Science and Information Systems, vol. 13, no. 2, pp. 427-452, 2016.

[3] C. Courtois and E. D'Heer, "Second screen applications and tablet users: Constellation, awareness, experience, and interest," in Proceedings of the 10th European Conference on Interactive TV and Video, EuroiTV '12, pp. 153-156, ACM, NY, USA, 2012.

[4] M. Ludvigsen and R. Veerasawmy, "Designing technology for active spectator experiences at sporting events," in Proceedings of the 22nd Conference of the Computer-Human Interaction Special Interest Group of Australia on Computer-Human Interaction, pp. 96-103, ACM, 2010.

[5] E. Anstead, S. Benford, and R. J. Houghton, "Many-screen viewing: Evaluating an olympics companion application," in Proceedings of the 2014 ACM International Conference on Interactive Experiences for TV and Online Video, TVX '14, pp. 103-110, ACM, NY, USA, June 2014.

[6] L. Barkhuus, A. Engström, and G. Zoric, "Watching the footwork: Second screen interaction at a dance and music performance," in Proceedings of the SIGCHI Conference on Human Factors in Computing Systems, CHI '14, pp. 1305-1314, ACM, NY, USA, 2014.

[7] M. Barnidge, T. Diehl, and H. Rojas, "Second Screening for News and Digital Divides," Social Science Computer Review, 2018.
[8] A. Van Cauwenberge, G. Schaap, and R. van Roy, “Tv no longer commands our full attention: Effects of second-screen viewing and task relevance on cognitive load and learning from news," Computers in Human Behavior, vol. 38, pp. 100-109, 2014.

[9] R. M. Costa Segundo and C. A. Saibel Santos, "Systematic review of multiple contents synchronization in interactive television scenario," ISRN Communications and Networking, vol. 2014, 2014.

[10] L. Cruickshank, E. Tsekleves, R. Whitham, A. Hill, and K. Kondo, "Making interactive TV easier to use: Interface design for a second screen approach," The Design Journal, vol. 10, no. 3, pp. 41-53, 2007.

[11] F. Giglietto and D. Selva, "Second Screen and Participation: A Content Analysis on a Full Season Dataset of Tweets," Journal of Communication, vol. 64, no. 2, pp. 260-277, 2014.

[12] R. C. Nee and D. M. Dozier, "Second screen effects: Linking multiscreen media use to television engagement and incidental learning," Convergence, vol. 23, no. 2, pp. 214-226, 2016.

[13] C. E. Valente and C. T. Souza, "Support architecture for second screen apps dynamic adaptation," in Proceedings of the 23rd Brazillian Symposium on Multimedia and the Web, pp. 365-372, NY, USA, 2017.

[14] H. Gil de Ziiga, V. Garcia-Perdomo, and S. C. McGregor, "What is second screening? exploring motivations of second screen use and its effect on online political participation," Journal of Communication, vol. 65, no. 5, pp. 793-815, 2015.

[15] M. Macik, E. Lorencova, Z. Mikovec, and O. Rakusan, "Software architecture for a distributed in-hospital navigation system," in Proceedings of the 2015 Conference on Research in Adaptive and Convergent Systems, RACS, pp. 369-375, ACM, NY, USA, 2015.

[16] S. Livingstone, "The challenge of changing audiences: or, what is the audience researcher to do in the age of the internet?" European Journal of Communication, vol. 19, no. 1, pp. 75-86, 2004.

[17] M. Deuze, "Media life," Media, Culture \& Society, vol. 33, no. 1, pp. 137-148, 2011.

[18] E. Tsekleves, R. Whitham, K. Kondo, and A. Hill, "Investigating media use and the television user experience in the home," Entertainment Computing, vol. 2, no. 3, pp. 151-161, 2011.

[19] P. Dalsgaard, C. Dindler, and E. Eriksson, "Designing for participation in public knowledge institutions," in Proceedings of the 5th Nordic Conference on Human-computer Interaction: Building Bridges, NordiCHI '08, pp. 93-102, ACM, NY, USA, 2008.

[20] M. Ebner and A. Holzinger, "Lurking: An underestimated human-computer phenomenon," IEEE MultiMedia, vol. 12, no. 4, pp. 70-75, 2005.

[21] B. Nonnecke and J. Preece, "Lurker demographics: Counting the silent," in Proceedings of the SIGCHI Conference on Human Factors in Computing Systems, CHI '00, pp. 73-80, ACM, NY, USA, 2000.

[22] T. Cerny and M. J. Donahoo, "MetamorPic: Self-contained photo archival and presentation," in Information Systems Development, pp. 149-158, Springer, NY, USA, 2001.

[23] L. DeMichiel and B. Shannon, JSR 342: Java TM Platform, Enterprise, 7th edition, 2013, https://jcp.org/en/jsr/detail?id=342.

[24] F. Vogelsteller, Building Single-page Web Apps with Meteor, Packt Publishing Ltd, 2015.

[25] R. Hanson and A. Tacy, GWT in Action: Easy Ajax with the Google Web Toolkit, Manning Publications Co., Greenwich, England, CT, USA, 2007. 
[26] E. Burns and N. Griffin, JavaServer Faces 2.0, The Complete Reference, McGraw-Hill, Inc, NY, USA, 1st edition, 2010.

[27] A. Freeman, Pro AngularJS, Apress, Berkeley, CA, USA, 1st edition, 2014.

[28] F. Marchioni, Infinispan data grid platform, Packt Publishing Ltd, 2012.

[29] J. L. Carlson, Redis in Action, Manning Publications Co, Greenwich, CT, USA, 2013.

[30] M. Raible, Spring Live, SourceBeat, LLC, 2004.

[31] B. Clifton, Advanced Web Metrics with Google Analytics, SYBEX Inc, Alameda, CA, USA, 2008.

[32] D.-G. Tremblay and V. Psyché, "Analysis of processes of cooperation and knowledge sharing in a community of practice with a diversity of actors," Computer Science and Information Systems, vol. 9, no. 2, pp. 917-941, 2012.

[33] T. Cerny and M. Donahoo, "Survey on second screen systems," in Proceedings of the 2016 6th International Conference on IT Convergence and Security (ICITCS), pp. 1-5, 2016. 


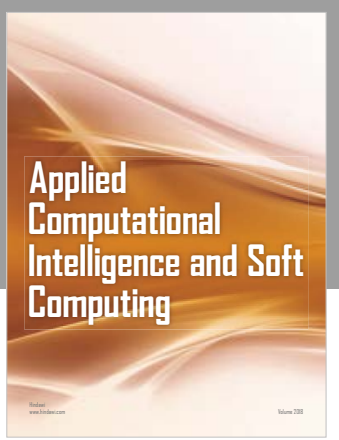

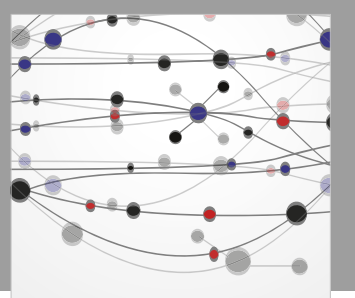

The Scientific World Journal
Submit your manuscripts at

Computing
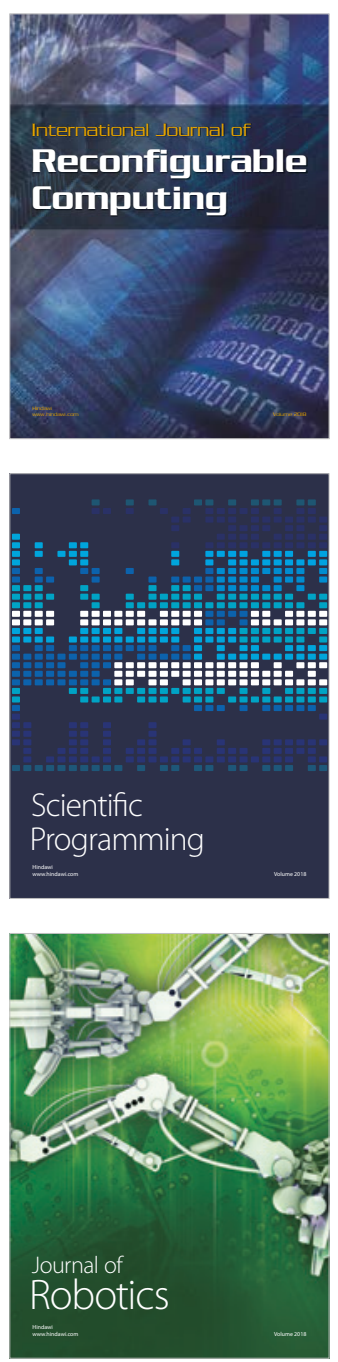

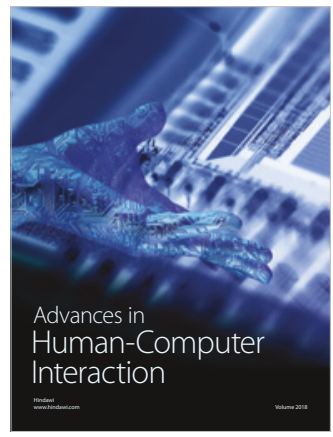

Human-Compute

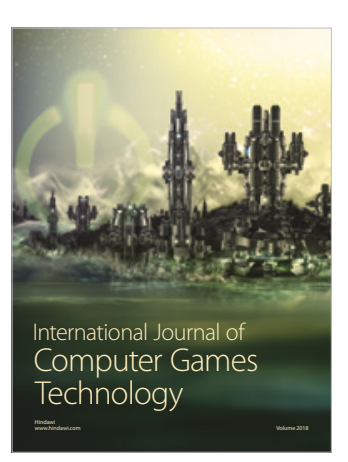

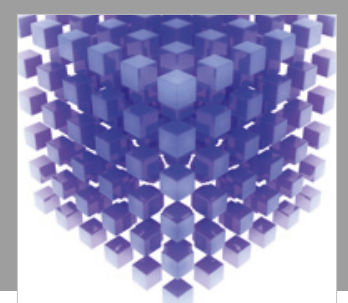

Mathematical Problems in Engineering

\section{Engincering}
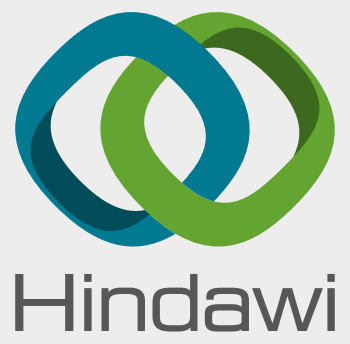

www.hindawi.com
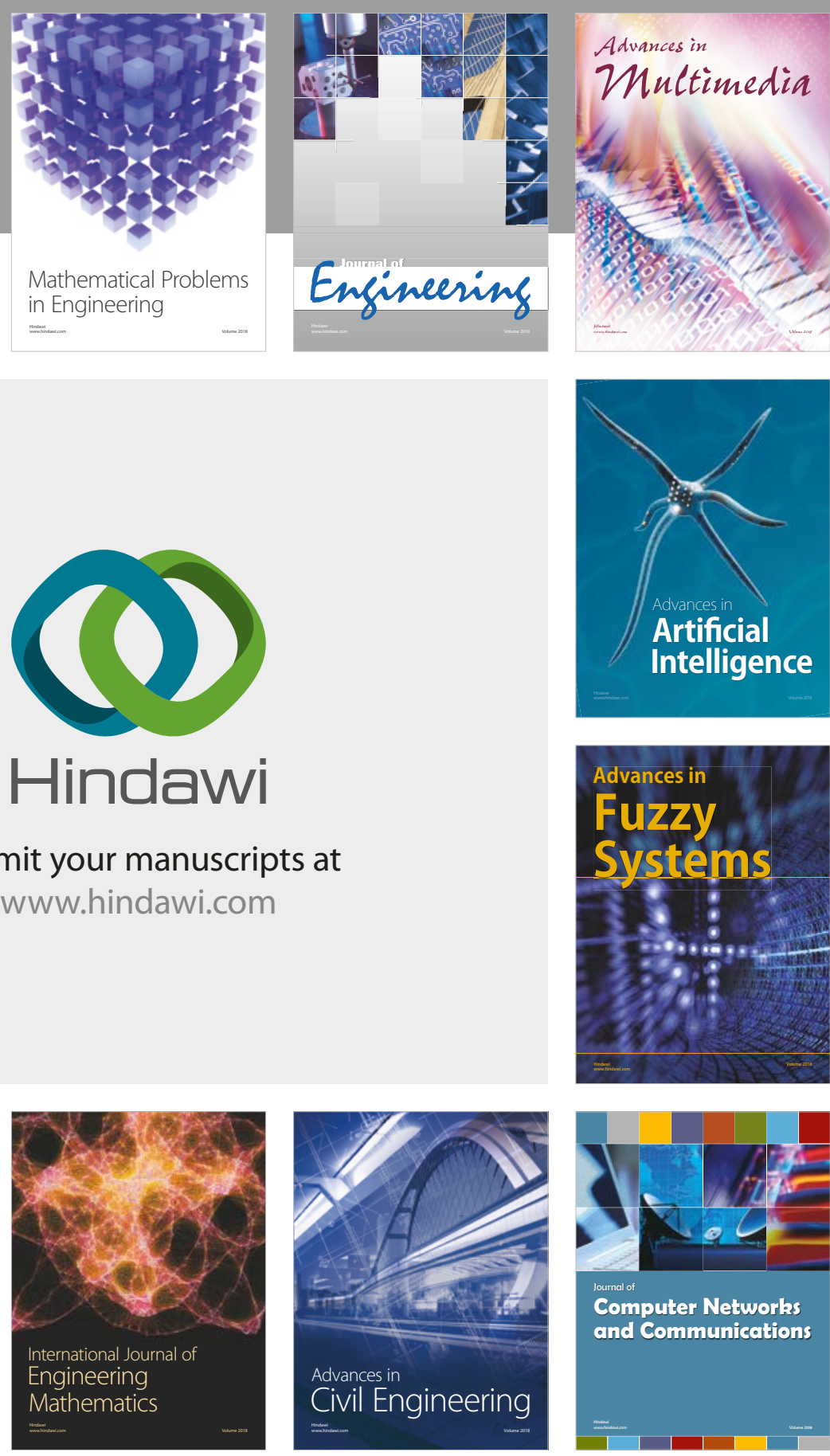

Computer Networks and Communications

Multimedia
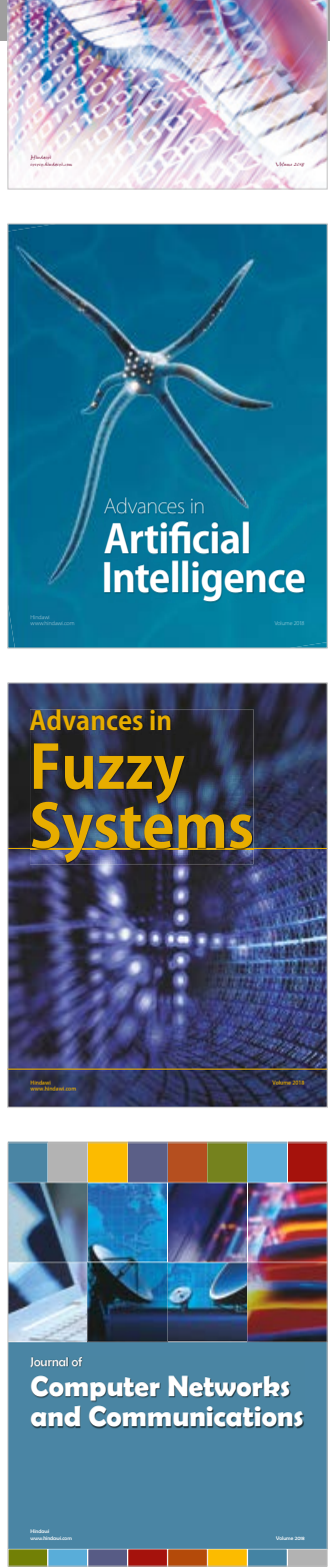

Advances in

Modelling \&

Simulation

in Engineering

interaction

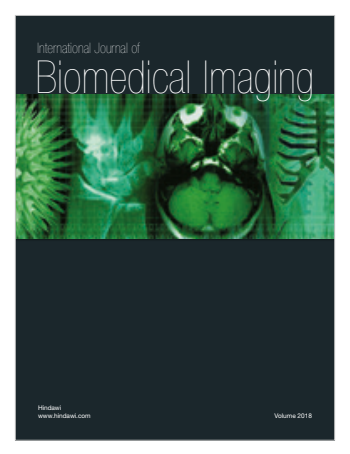

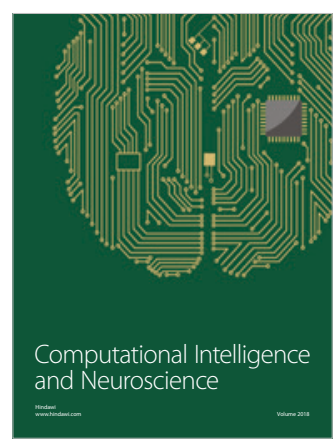

